\title{
Parametric Structural Analysis for the SpaceLiner
}

\author{
A. Kopp ${ }^{1}$ and N. Garbers ${ }^{2}$ \\ German Aerospace Center (DLR), Space Launcher Systems Analysis (SART), Bremen, 28359, Germany \\ and \\ R. Jarlas ${ }^{3}$ and H. Rabia ${ }^{4}$ \\ Swedish Defence Research Agency (FOI), Aeronautical systems, Stockholm, SE-164 90, Sweden
}

The Space Launcher Systems Analysis Group (SART) of the German Aerospace Center DLR has been working for several years on developing a novel hypersonic passenger transportation concept. The SpaceLiner, originally proposed in 2005 , is a two staged, rocket propelled and vertical take-off transportation system designed for a 90 minutes EuropeAustralia reference mission carrying 50 passengers. In addition to the DLR internal activities, the SpaceLiner is also under investigation in the current European Commission's (EC) research projects FAST20XX and CHATT. A major challenge for the SpaceLiner is the design of a lightweight structure for this unique vehicle concept and the integration of structure and thermal protection system (TPS). For this task, a finite element based structural analysis tool will be used, providing rapid parametric studies for complex vehicle configurations with a high level of flexibility. This paper summarizes the current and past activities on the preliminary structural analysis for the SpaceLiner. The parametric analysis tool will be described in detail. Different structural concepts will be investigated and the results presented. Also discussed is the integration of TPS and- structure, as well as structural-dynamic considerations. In addition to the SpaceLiner related activities also a brief summary of the parametric structural analysis within the EC co-funded ATLLAS-II research project will be given.

\begin{tabular}{ll} 
& \multicolumn{1}{c}{ Nomenclature } \\
AFRSI & $=$ Advanced Flexible Reusable Insulation \\
ATLLAS & $=$ Aero-Thermodynamic Loads on Lightweight Advanced Structures \\
$C H A T T$ & $=$ Cryogenic Hypersonic Advanced Tank Technologies \\
$C M C$ & $=$ Ceramic Matrix Composite \\
$C R I$ & $=$ Conformable Reusable Insulation \\
c. . $g$. & $=$ Center of Gravity \\
$d \nu$ & $=$ Design Variable \\
$E C$ & $=$ European Commission \\
$F A S T$ & $=$ Future High-Altitude High-Speed Transport \\
$F E A$ & $=$ Finite Element Analysis \\
$F O I$ & $=$ Totalförsvarets Forskningsinstitut (Swedish Defence Research Agency) \\
$G D L$ & $=$ Gas Dynamics Ltd \\
$G L O W$ & $=$ Gross Lift Off Weight \\
$M$ & $=$ Mach Number \\
$M E C O$ & $=$ Main Engine Cut-Off \\
$M P C$ & $=$ Multi Point Constraint \\
$P M P$ & $=$ Propellant Management Program
\end{tabular}

\footnotetext{
${ }^{1}$ Research Scientist, Space Launcher Systems Analysis, DLR, Member of AIAA.

${ }^{2}$ Research Scientist, Space Launcher Systems Analysis.

${ }^{3}$ Senior Scientist, Information- and Aeronautical Systems, FOI.

${ }^{4}$ Senior Scientist, Information- and Aeronautical Systems, FOI.
}

American Institute of Aeronautics and Astronautics 


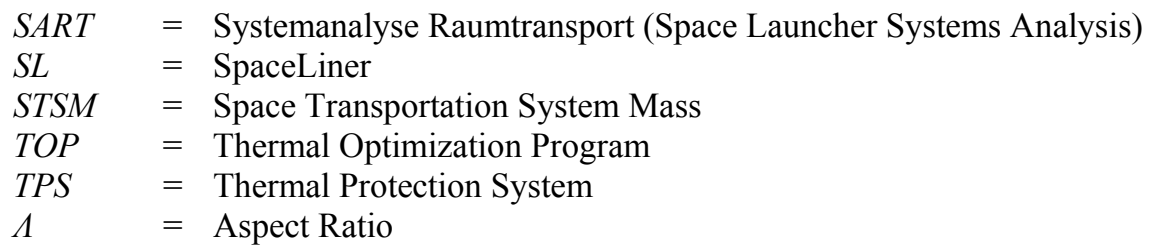

\section{Introduction}

$\mathrm{T}$ HE two-staged rocket propelled SpaceLiner concept has been proposed for enabling ultra-fast antipodal passenger transport ${ }^{1,2,3,4}$. The system launches vertically and consists of a passenger-carrying, so called "orbiter" stage, and a booster stage. Both stages are fully reusable and utilize liquid propellants. After main engine cut-off (MECO) of the booster stage, the latter is separated and returns to ground. The orbiter then continues the ascent with its own rocket propulsion system. After having burnt all propellants, the orbiter begins hypersonic range flight in a gliding mode without additional propulsion. The reference mission carries 50 passengers from Europe to Australia within 90 minutes and vice-versa. Other missions and destinations are also under investigation.

In contrast to other hypersonic transportation concepts, the SpaceLiner does not incorporate radically new or unproven technologies. Nevertheless, the concept contains a number of technical and logistical challenges, such as active cooling systems, passenger accommodation and safety, or the provision of suitable launch and landing sites.

A further crucial challenge is the design of a lightweight structure. In fact, the vehicle is highly mass critical and the design of a lightweight structure is not only a performance issue, but may even determine the concept's feasibility. The structural analysis is complicated due to the very specific passenger accommodation within a separable rescue capsule. This design has been incorporated to account for the comparatively low reliability of rocket launcher systems when compared to conventional turbofan propelled passenger aircraft. In the case of a critical emergency, the passenger capsule will be ejected from the main stage to bring the passengers back safely to the ground. Part a) of Fig. 1 shows an artist's impression of the SpaceLiner at booster separation and part b) an early design of the rescue stage.

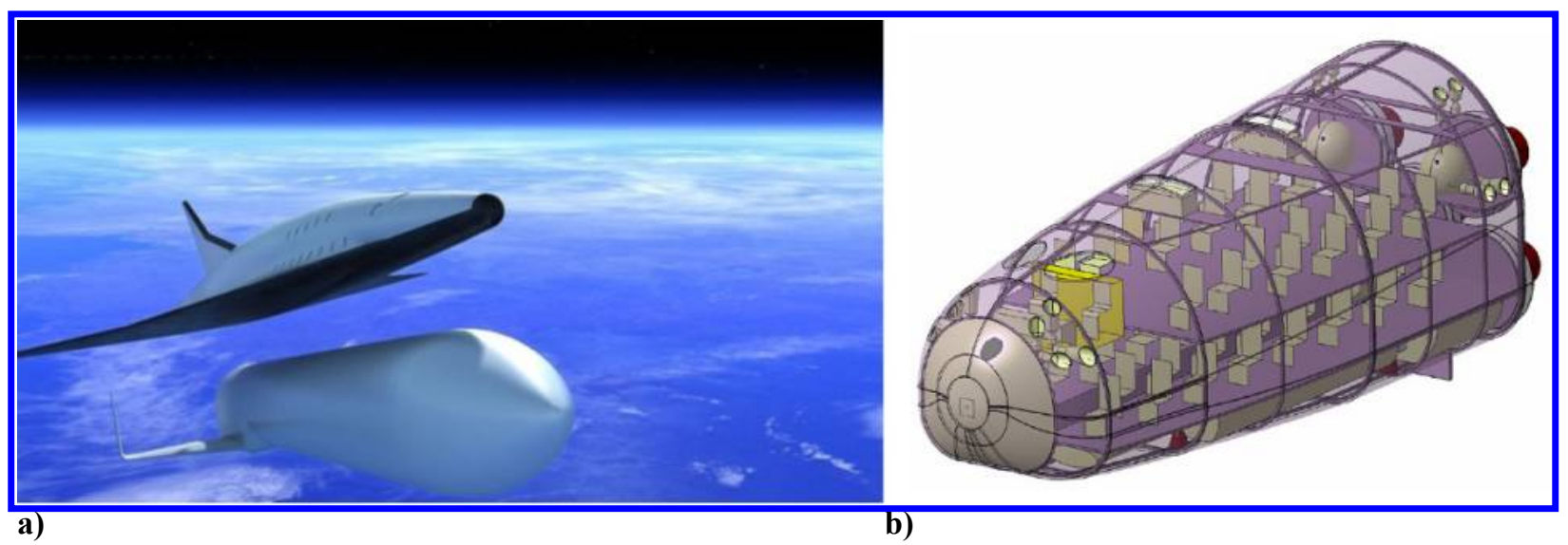

Figure 1. SpaceLiner2 at booster separation a); early design of the rescue stage b).

MECO of the orbiter stage occurs at about $70 \mathrm{~km}$ altitude with a velocity of more than $7 \mathrm{~km} / \mathrm{s}$. The subsequent gliding trajectory profile of the orbiter with Mach numbers well beyond 20 requires very thick thermal protection systems. Thus, the integration of TPS and structure while still assuring a low structural mass presents a further, major challenge.

The current design of the SpaceLiner, the SpaceLiner7 (SL7), is the latest version of a series of evolving designs, beginning with the SpaceLiner1 in 2005. In all previous configurations only empirical/statistical relationships had been employed to estimate the structural weight. The SpaceLiner7 stands alone as the first configuration for which structural analyses are performed. Also, the SL7 is the first vehicle which incorporates a comparatively detailed, but still provisional TPS design, while very preliminary TPS investigations had already been performed for the SL4.

This paper focuses on the structural design of the orbiter stage. Structural investigations for the booster stage are currently being performed at FOI, but will not be discussed in this paper. 


\section{Structural Analysis Trade Study Aims}

For vehicle system studies on a preliminary level, with rapidly changing configurations, it is required to produce comparatively reliable structural mass data in order to evaluate the vehicle performance characteristics. In particular, for vehicles concepts with very low performance margins, the structural mass may decide if a vehicle concept and its mission is even feasible. At a preliminary design level, usually statistical/empirical methods are utilized for structural mass estimation. Structural analysis computations follow only at an advanced design phase. This often is an appropriate approach because statistical/empirical generated mass data are often more reliable than the results of calculations with simple structural analysis programs combined with preliminary structural designs, being far from optimized.

However, this approach also has its disadvantages. This is particularly true if the vehicle concepts being investigated are unique in shape, configuration or mission, and do not find any equivalents in the statistical data base. As an example, it is not uncommon for new vehicle concepts, evaluated by different design teams, to differ in estimated structural mass by $50 \%$ or even up to $100 \%$. Furthermore, the use of these statistical/empirical tools often does not allow for the identification of tendencies or the impact of special design features, which may be of major importance for the vehicle design process. Finally, preliminary design of advanced aerospace vehicles regularly requires estimations of structural thicknesses, especially if the structure is to be combined with a thermal protection system and/or the vehicle is highly volume critical. Thus, it is often necessary to apply structural analysis tools at an earlier design stage than common for more traditional vehicles.

For the current preliminary design of the SpaceLiner7 the structural discipline has to contribute the following points:

1) Estimation of TPS thicknesses

2) Definition of materials and structural concepts

3) Preliminary definition of main structural member positions

4) Initial structural sizing; structural dimensions, thicknesses

5) An estimation of the structural mass

The first point is of major importance, as the SpaceLiner orbiter internal volume is a highly critical parameter, and the TPS thicknesses computed in previous investigations have been found to be very high ${ }^{5,6}$. In fact, it was found that the TPS thickness alone already consumes a major part of the construction height of the wing, particularly in the wing tip regions. Thus, the profile thickness in the wing tip regions had to be increased, which in turn slightly lowered the Lift/Drag ratio. This highlights the importance of TPS and structural thickness estimations already at early design stages.

Furthermore, early material trade-off studies and selections are important not only for structural considerations. The material selection has also a strong influence on the required thickness of the TPS, as higher allowed backstructure temperatures may reduce the demands for the TPS.

\section{Structural Analysis Approach}

At the current stage of the system design process, a structure that is light-weight as well as thin in terms of construction height, is envisaged. A structural concept shall be designed that is able to provide the feasibility of the vehicle and its mission. It is not the aim of the current study to optimize the structural design. In fact, it is assumed that the design of an optimal structural concept requires larger resources than available at this stage. It is not appropriate, nor practical, to perform an optimization in the middle of an iterative design process. To find a suitable structural design, parametric studies are being conducted. These include variation of structural materials and construction concepts, as well as the repositioning or re-spacing of the main structural members.

As is usual in preliminary system analysis, tools are required that are suited for parametric studies with rapidly changing configurations while providing reasonable accurate results with low model-creation and calculation times. Comparatively simple, analytical tools are typically used for this task. For hypersonic vehicles however this class of tools may be less suited, as geometries and internal designs of hypersonic vehicle concepts may be complex and unique. Figure 2 shows different vehicle concepts under investigation in DLR-SART or within EC research projects with DLR-SART involvement. Representatives for different classes of high speed transportation vehicles are displayed. This includes configurations with conventional wing/body layouts, more complex waverider-shaped designs or air-breathing vehicles with a high level of integration of propulsion system and structure. Typical challenges for structural analysis include propellant tank design and integration in complex vehicle shapes, propulsion and thermal protection system (TPS) integration, and in general, efficient and light-weight structural 
design for vehicles shapes with poor conditions from a structural-mechanics point of view (e.g. low construction height, large surface-to-volume ratios).

To cover these different concepts, with high levels of geometric complexity, it is appropriate to apply numerical methods rather than analytical ones. By doing this, it is possible to take advantage of the rapid decrease in computer calculation times in recent years together with improved parametric modelling capabilities of modern finite element analysis (FEA) software. In particular, the second point is of major importance, since parametric modelling and structural analysis of arbitrary and rapidly changing vehicle configurations poses a significant challenge for analytic tools, while comparatively simple when using parametric FE methods. Especially the ANSYS Parametric Design Language

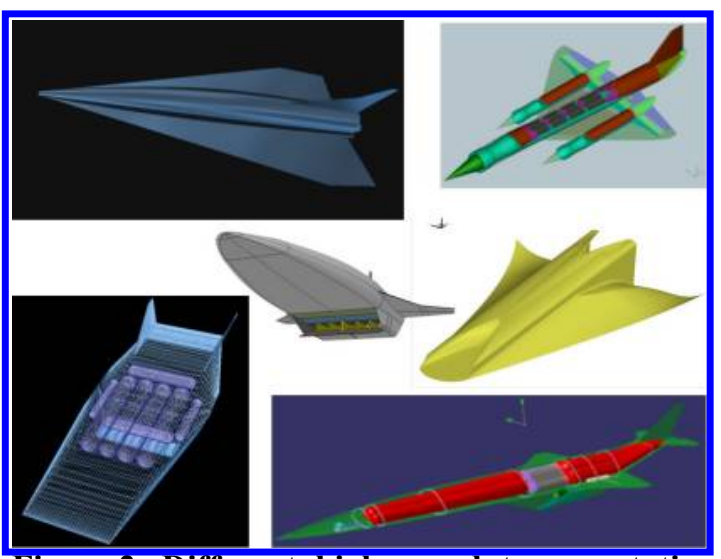

Figure 2. Different high speed transportation vehicle designs with DLR-SART involvement. (APDL) provides an excellent environment for fast parametric modelling of geometrically complex structures.

\section{A. HySAP - Hypersonic Vehicle Structural Analysis Program}

According to the requirements, as derived in the previous section, the Hypersonic vehicle Structural Analysis Program (HySAP) has been developed. Its main task is to perform rapid parametric structural analysis on a preliminary design level for almost arbitrary vehicle configurations, with low modelling and calculation times. HySAP combines Fortran pre-processor and Fortran sizing routines with the ANSYS Mechanical program system. The application of Fortran as programming language enables the use of existing routines from other structural analysis tools used within SART. The development of HySAP is not finished, but an initial version of the program is being used for the current investigations.

\section{Program Structure}

The Fortran based pre-processor HySAP creates an APDL input file for ANSYS. This file contains all commands for geometry generation, loads application, meshing, solution and post-processing as well as iteration step and load case information. Once launched, ANSYS is able to automatically perform all operations as provided in the input file. The structural sizing will be done outside ANSYS with the help of a separate Fortran based sizing tool. This tool is called by ANSYS after determining a solution. The sizer validates the structure against several strength and stability failure modes and adapts wall thicknesses as necessary. ANSYS then restarts the modeling and computation process with the adapted wall thicknesses. This procedure is repeated several times and for several load cases until convergence, or until a user defined maximum number of iterations, has been reached. No user intervention is required during the whole process. Figure 3 shows the general program organization. Four different operation modes are available. In the first, only geometry modelling is performed. This gives the user the opportunity to visually inspect the vehicle and to rearrange the geometry, if necessary. In further modes, the geometry meshing, the computations, and finally the complete

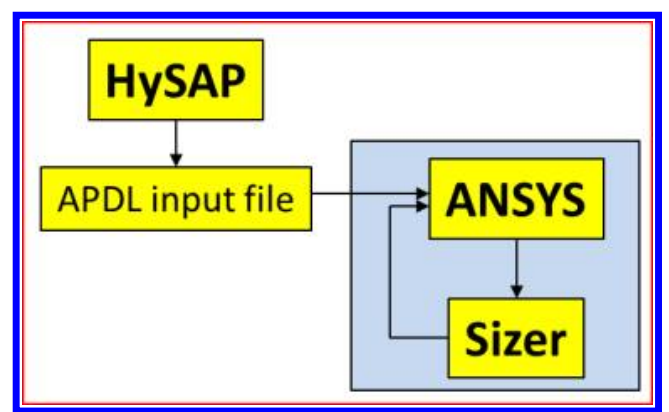

Figure 3. General program organization of HySAP. iteration cycle, are added.

\section{Input Processing}

HySAP is connected to other system analysis tools available in DLR-SART in order to receive input data from the particular disciplines. Aerodynamic pressure distributions as well as the surface mesh are provided by the DLRcode Hotsose. Hotsose generates hypersonic aerodynamic data sets by using inclination based methods. A panel code derived from the NASA program PanAir ${ }^{7}$ is planned to be connected to HySAP as well, in order to provide low speed pressure distributions. Propellant tank geometry, propellant mass, and pressure data, will all be generated by the SART PMP (Propellant Management Program) tool, while subsystem masses and center of gravity (c.o.g) positions will be provided by STSM (Space Transportation System Mass). Furthermore, the 1D thermal analysis 
code TOP2 will be connected to HySAP to estimate TPS thicknesses in the future. At the moment, all integration of structure and TPS has to be performed manually.

User defined load cases will be read from a separate file. Since vehicle concepts, missions and load environments may be unique, it is not appropriate to automate this process and provide a number of standard load cases, as it is often done in conventional transportation aircraft preliminary structural analysis.

For each load case, accelerations or the utilization of inertia relief capability have to be specified, as well as the use of the present aerodynamic pressure distribution. If fixed accelerations are imposed rather than inertia relief, the mounting conditions have to be specified. This is simply done by providing the subsystem(s) identification number(s), where the vehicle is to be mounted on (e.g. the main gears for a landing load case or the main engine for a vertical lift off case).

Structural data and material selections have to be provided by the user as well. This includes data such as rib, spar of frame positions, skin or web stiffening concepts, and initial wall thickness. Materials may be selected for structural groups such as wing ribs, wing spars, wing skin etc. separately.

FEA specific parameters generally do not have to be specified by the user. This allows also users with limited FEA experience to apply the program. The only exception is the average element size, which can be adjusted in order to control the calculation times and the fidelity of the results.

\section{Geometry Modelling}

The vehicle outer mould line as provided by the aerodynamic mesh is segmented in components (e.g. fuselage, wings, fin etc.). This segmentation will be taken over by HySAP. The aerodynamic mesh is transformed to an ANSYS geometry mesh with lower resolution, which may be seen in Fig. 4. The geometry contour will be defined by rib-spar or frame/bulkhead-skin junctions. Key points will be generated at these junctions. Additional contour definition key points will be generated automatically, as necessary, in order to better match the aerodynamic contour. With the help of the key points defined, areas will be generated such as rib, spar or skin areas. Initially, wing and fin components will be modelled. Ribs and spars will be generated as indicated in the input file. Additional spars will be generated automatically at the rib/leading edge junctions. Subsequently, the fuselage is modelled and the wings and fins attached. Frame/bulkhead stations will be created at user defined positions and additionally at wing-fuselage or fin-fuselage spar attachment points. The bulkheads will transmit wing bending loads though the fuselage rather than wing-box carry-through constructions. The latter type of load transmission is not yet available in HySAP since hypersonic vehicles usually demand large, fuselage-mounted and pressurized propellant tanks that cannot be intersected by a carry-through. After fuselage generation, the propellant tanks will be modelled. Cylindrical and conical tanks in single- and multi-lobe designs can be modelled (shown in Fig. 5). The geometry is entirely read from PMP output files. Bulkhead-tank intersections will be considered with Boolean operations and corresponding cut-outs in the bulkheads will be generated. Currently, the bulkheads are completely connected to the tanks. Consequently, they support the fuselage in carrying longitudinal bending loads. Alternative mounting concepts are planned for introduction in further modifications. All main structural members such as ribs, spars, bulkheads or skins are defined by areas. All of the areas will later be meshed with shell elements. Stiffening of the areas may be

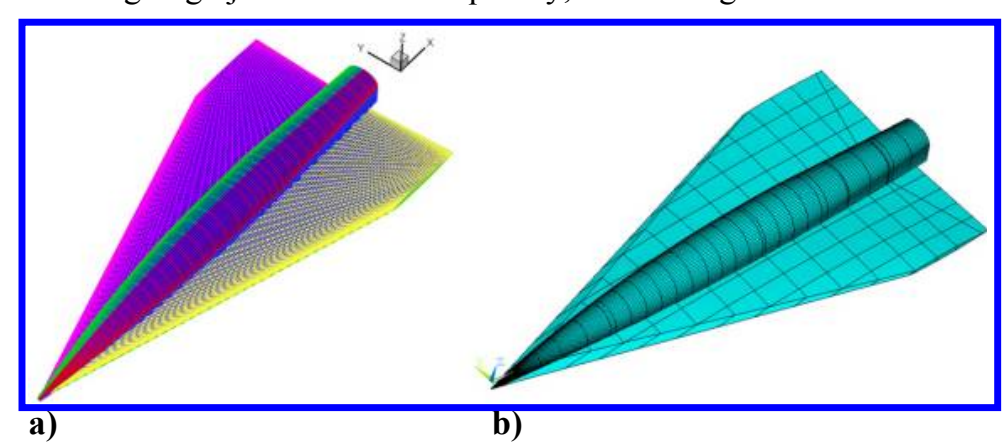

Figure 4. Aerodynamic mesh a); ANSYS geometry mesh b).

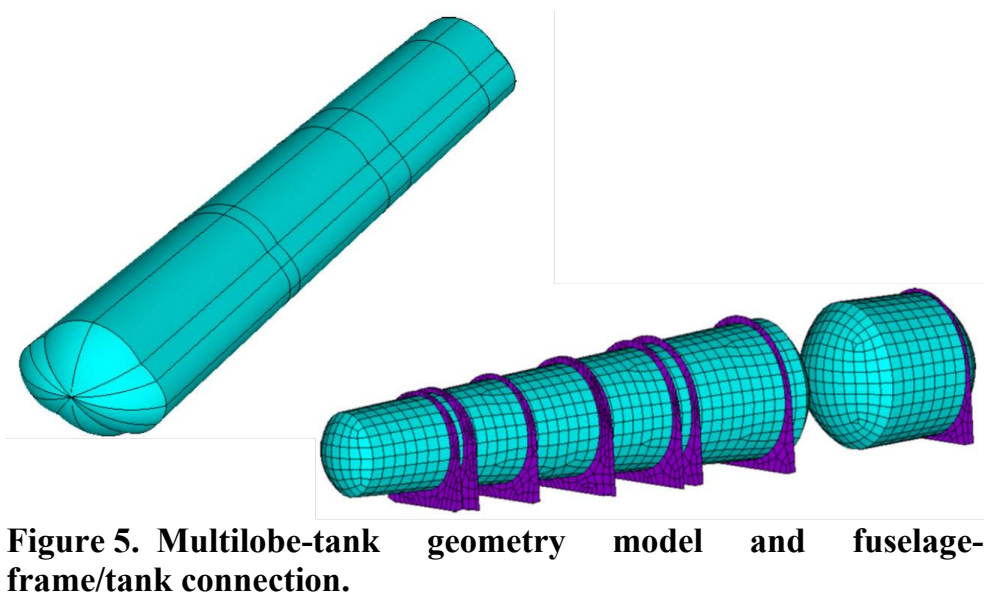

American Institute of Aeronautics and Astronautics 
selected by the user. Generally, stiffening is modelled with a "smeared" approach, as often done in preliminary analysis. Currently, unstiffened-, uni-axial stringer stiffened- and sandwich panels can be considered. This is done internally by assigning multiple layers to each area. In the case of stringer stiffening the first layer is the skin, while the second layer represents the smeared stringer layer. An approach derived from ref. 8 and ref. 9 has been applied to model the stringer layer. For sandwich materials three different layers will be modelled, with the middle layer as the sandwich core.

\section{Loads Modelling}

Several groups of loads may be applied: aerodynamic pressures, tank static and hydrostatic pressures, and accelerations, which yield inertia loads. The latter includes the loads from the structure itself, as well as from subsystem masses. Finally, user-defined point loads or moments can be introduced.

Propellant static pressures will be read from PMP files and applied to the internal surfaces of the tanks. Pressurized passenger cabins may be also modelled in this way. Additionally, hydrostatic pressures will be computed according to the present accelerations and the propellant masses. The corresponding fluid surface positions and attitudes will be computed correctly as shown in Fig. 6. Subsystem masses and positions will be read from STSM output files. In HySAP, all subsystems will be modelled as mass points. The introduction of their inertia load is realised with rigid and mass-less Multi-Point-Constraint (MPC) elements. Fuselage subsystems will be attached to the nearest forward and aft bulkhead/frame with several MPC's. Wing systems such as gears instead will be connected to up to four rib/spar junctions. Fig. 7 shows subsystem mass points with their MPC attachments for a vehicle configuration. The vehicle is seen from the top and the structure has been removed in this figure. The most upper and lower mass points represent landing gears accommodated in the wings.

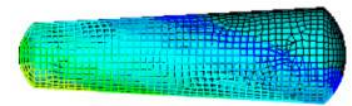

Figure 6. Hydrostatic tank pressures due to normal and axial accelerations.

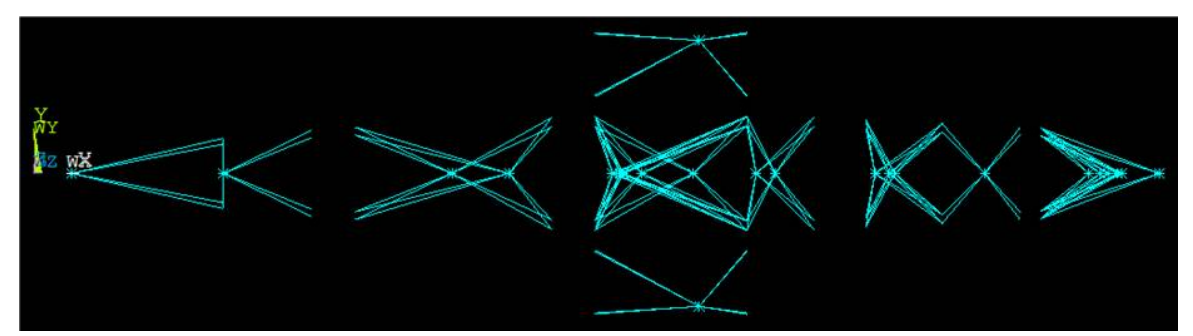

Figure 7. Sub-system attachment via MPC's.

\section{Meshing}

One, two or three-layered shell elements, depending on the particular stiffening type, will be used for the complete model. The only exceptions are the MPC and point elements for subsystem mass definition. The meshing scheme is not predefined due to the complex geometry. Instead, the "free meshing" capability of ANSYS is exploited. The average element size can be specified by the user, thus allowing for computation time and accuracy control. ANSYS is instructed by HySAP to use quadrilateral shell elements for meshing, whenever possible. A small number of trilaterals is unavoidable. The automated mesh generation procedure has been found to be very stable for complex configurations, as long as the average elements size is not too large. Figure 8 shows a corresponding vehicle model with different mesh sizes.

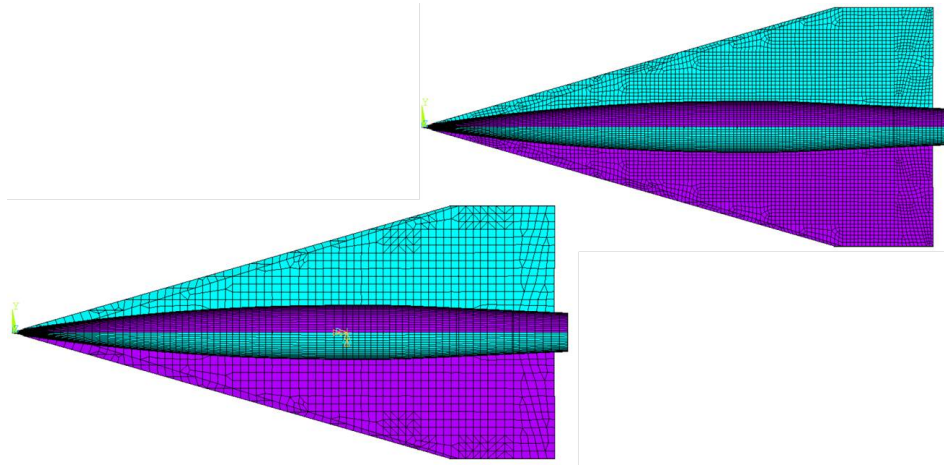

Figure 8. Surface meshes with different average element sizes.

\section{Structural Analysis and Sizing Strategy}

Structural sizing is done by a separate Fortran-based sizing tool. After every iteration step the sizer will be called by ANSYS and performs the structural sizing. In the next iteration step, ANSYS will apply the adapted wall thickness to the structure. The data exchange between ANSYS and the Fortran binary has been realized on a file basis. 
The structure is divided in 'optimization components'. Each of these components will be sized individually and assigned a uniform wall thickness. For wings, each geometry area constitutes an optimisation component, which means that each skin, rib, or spar panel will be sized individually. For fuselages and tanks, the whole section between two frame/bulkhead stations is defined as optimisation component and assigned a uniform thickness. This approach may lead to conservative structural mass estimations of fuselages and will be adapted in further program modifications.

In the current program version the structure will be sized based on the following design allowables:

1) Von Mises stress (all components)

2) Sheet buckling for unstiffened panels as derived from ref. 10

3) Stringer flexural and stringer local buckling as derived from ref. 8 and ref. 9

4) Sandwich panel global buckling as derived from ref. 11

5) Sandwich panel wrinkling as derived from ref. 12

These allowables are the basis for initial structural mass estimations. Additional design criteria will be implemented in the future to enable more accurate mass computations.

A minimum gauge thickness is being applied to skins, sandwich face-sheets and stringer thicknesses. Typical values may be in the range of $0.25 \mathrm{~mm}-1 \mathrm{~mm}$. For instance, ref. 13 suggests a minimum gauge thickness of $0.25 \mathrm{~mm}(0.01 \mathrm{in}$.) for isotropic materials, while proposing higher values for composites.

No optimization procedure is implemented so far and only skin and stringer thicknesses will be varied during the iteration process. Heights of stringer layers or sandwich cores remain constant and have to be predetermined by the user. This approach will be complemented by more sophisticated adaption methods in the future. The process of sizing each optimization component individually will not ensure the determination of any global optimum. However, critical evaluation of the results of previous program applications always yielded reasonable program behaviour and mass estimations.

\section{Parametric Structural Analysis within the ATLLAS-II Project}

The first application case of the HySAP program was within the European Commission co-funded research project ATLLAS-II (Aero-Thermodynamic Loads on Lightweight Advanced Structures II). This project is the successor of the completed ATLLAS-I study and, as was done in ATLLAS I, is focused on advanced structures and materials for future high-speed civil transportation vehicles. A total of 13 partners from six European countries are part of the ATLLAS-II consortium. The total budget is 6.5 Mio $€$ with a project duration of 4 years.

Although, the ATLLAS project has no connection to the SpaceLiner, the parametric structural analysis as performed within ATLLAS will be discussed here briefly, to demonstrate the benefit of this kind of analysis in early vehicle design.

Part of the investigation within the project focused on the design of a reference configuration, which will be able to transport 200 passengers over intercontinental distances with a cruise speed of $\mathrm{M}=5-6$. Gas Dynamics Ltd (GDL/UK) designed three initial configurations with a special emphasis on aerodynamics and propulsion performance, while the Swedish Defence Research Agency FOI and DLR-SART are performing structural investigations. Figure 9 shows the three configurations, whereas the final reference configuration will be based on the so-called under-expanded/triple configuration ${ }^{14}$, the leftmost one in Fig. 9.

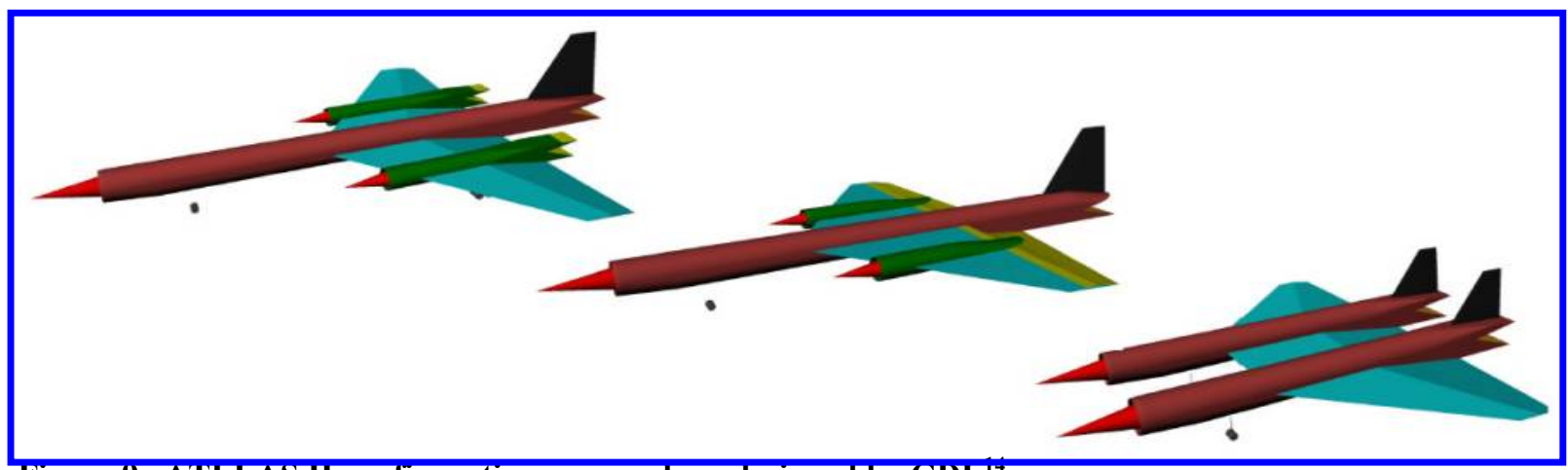

Figure 9. ATLLAS 11 configuration proposals as designed by GDL ${ }^{*}$. 
The structural investigations include first order trade-off studies for structural details such as wing carry-through or inlet flow channel integration, as well as low level detailed structural analyses for the complete configuration. The results of all these activities will be fed into an integrated optimization loop, which will finally yield the reference configuration.

At the time of the presented investigations within ATLLAS II the HySAP tool was still in development. A preliminary version was available and had been applied. Thus, the program was used for structural trade studies on a relative basis, rather than producing concrete and reliable mass data. Therefore, it was assumed that inaccuracies in the structural mass estimations would be similar for all investigated configurations, thus enabling relative comparisons between the configurations and the identification of trends.

One of the main subjects of these analyses was to investigate the impact of the wing aspect ratio on the structural weight. In fact, the aspect ratio was a free parameter from an aerodynamic cruise performance perspective, while the net wing area as well as wing profile and inclination angle remained fixed. Five different aspect ratios $\Lambda$, from 3.13 (initial design) down to 1.0 were analyzed, depicted in Fig. 10.

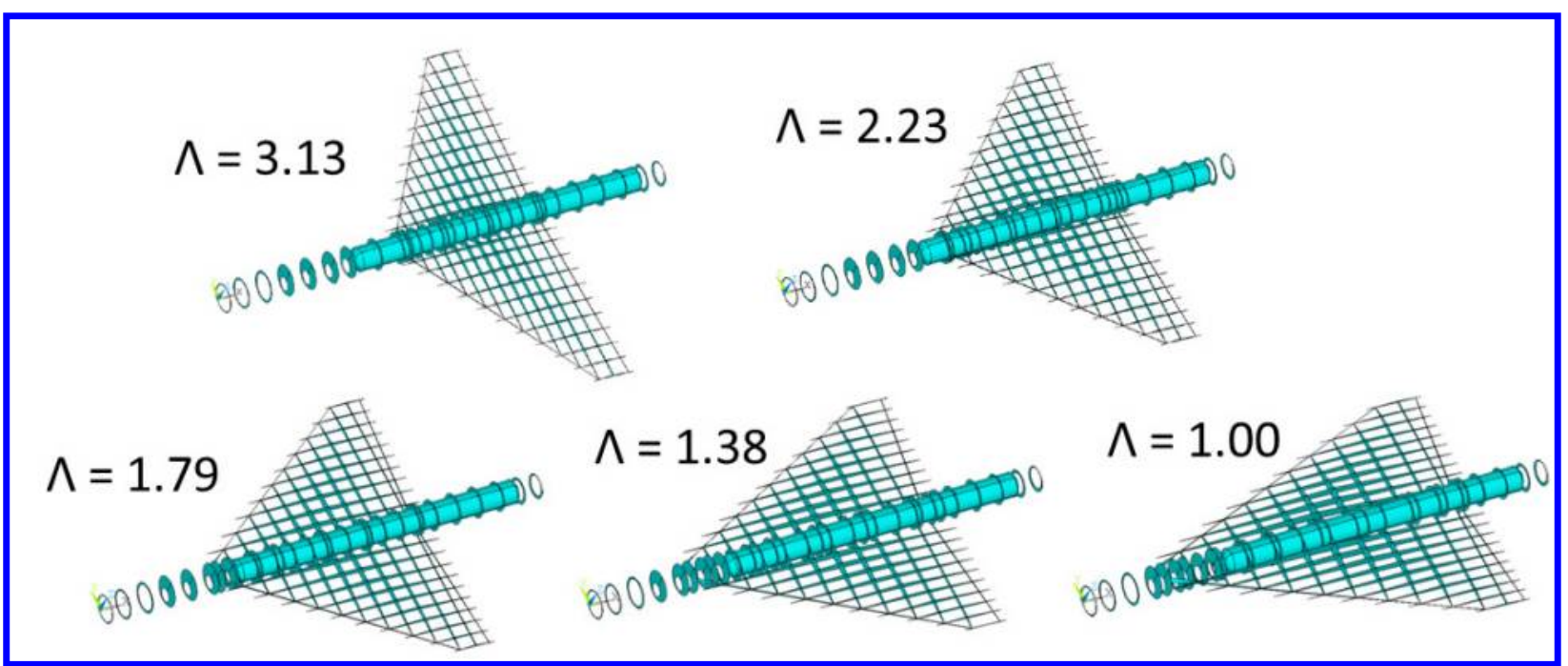

Figure 10. ANSYS geometry models of investigated configurations with different aspect ratios (skin areas removed in the figure).

The under-expanded/triple configuration (Fig. 9) has been selected as the study vehicle, whereas the wing-poded engine housings have been omitted in the analysis in order to reduce the complexity of the models. Two load cases have been considered successively:

1) Maneuver with $2.5 \mathrm{~g}$ normal acceleration and full propellant tanks

2) Maneuver with $2.5 \mathrm{~g}$ normal acceleration and empty propellant tanks

For the second load case a lower angle of attack was required to generate the same normal acceleration. Since the non-cryogenic hydrocarbon fuels were stored mainly in the wing, de-loading the latter, the second case was assumed to be more critical. The computational results revealed, that a minimum in structural mass could be reached between aspect ratios of 1.8-1.4 (Fig. 11). Interestingly it was found, that for lower aspect ratios only slight reductions in the wing mass could be achieved. Instead, the majority of the mass reduction resulted from a reduction of the fuselage mass, caused by the stiffening effect of deeper wing root chords and higher profile heights. Indeed, this result could not have been found by applying available empirical/statistical tools, since they usually consider wing and fuselage independently. These findings were integrated in the reference definition process and yielded a preliminary configuration with an aspect ratio of 1.67, as displayed in Fig. 12. 


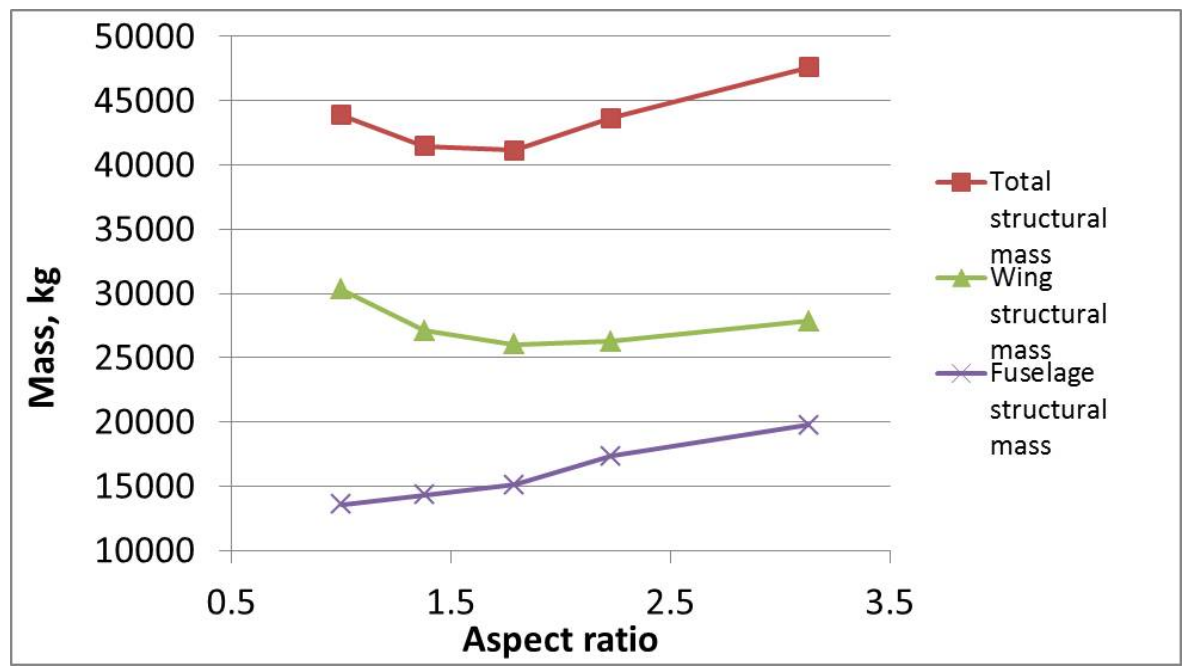

Figure 11. Computed structural masses for different aspect ratios.

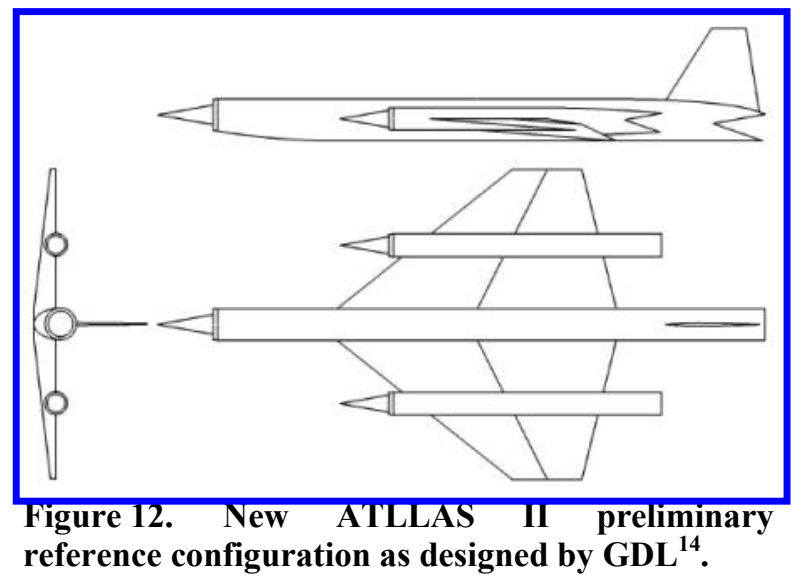

\section{Structural Analysis for the SpaceLiner}

As outlined in the previous sections, the main aim of the current analyses for the SpaceLiner is the identification of tendencies, rather than production of concrete mass values. Main subjects of these investigations are:

1) Variation of stiffening concepts

2) Variation of stiffener layer thicknesses

3) Variation of materials and impact on TPS mass

4) Variation of minimum gauge thicknesses

5) Impact of TPS thicknesses on structural mass

6) Impact of different load cases

The stiffening options are currently limited to unstiffened, uni-axial stringer stiffened, and sandwich concepts. The assessment of stiffener layer thickness variations is of major importance, since the vehicle is highly volume critical. The selection of different materials has to be considered together with the corresponding TPS mass, since higher temperature materials may allow for thinner TPS thicknesses.

\section{A. Configuration Description}

Figure 13 shows the latest design of the SpaceLiner7 with the orbiter attached to the booster. The main geometry and mass data can be found in Table 1. The total lift off mass of the system has a total of $1862 \mathrm{Mg}$, slightly lower than that of the Space Shuttle at $2050 \mathrm{Mg}$. Both stages utilize LOX and LH2 as propellants and all tanks are nonintegral. The most forward vessel within the orbiter represents the separable passenger rescue stage. This capsule is 
partly integrated in the fuselage of the orbiter. The upper part of the capsule also serves as the outer structure of the orbiter (Fig. 14). The lower half of the capsule instead forms a separate structure, encapsulated in the fuselage of the orbiter.
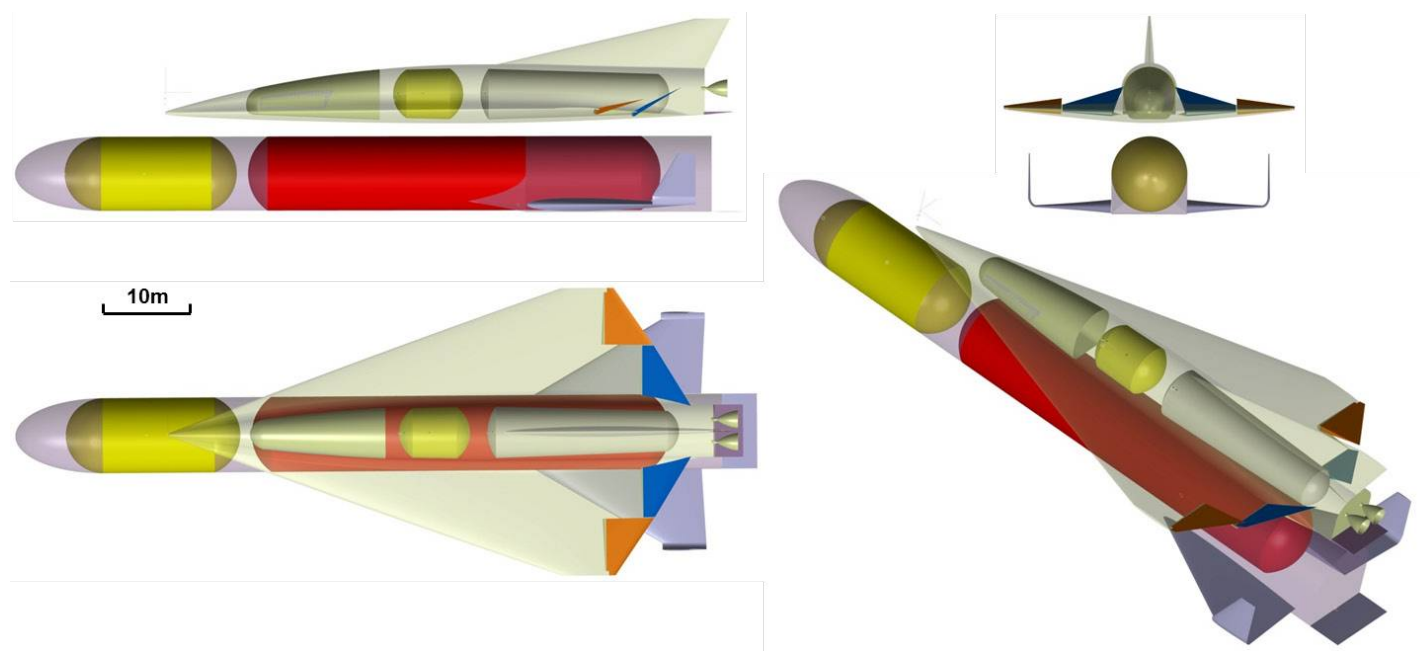

Figure 13. SpaceLiner7 booster and orbiter.

Table 1. Main geometry and mass data for the SpaceLiner7.

\begin{tabular}{|c|c|c|}
\hline & Orbiter & Booster \\
\hline Total length [m] & 65.6 & 83.5 \\
\hline Wing span [m] & 33.0 & 26.5 \\
\hline Height [m] & 12.1 & 8.7 \\
\hline Fuselage diameter [m] & 6.4 & 8.6 \\
\hline Dry mass [Mg] & 148.4 & 191.3 \\
\hline Payload mass [Mg] & 5.4 & - \\
\hline Propellant mass [Mg] & 218.5 & 1289 \\
\hline Total GLOW [Mg] & 377.6 & 1454.8 \\
\hline
\end{tabular}

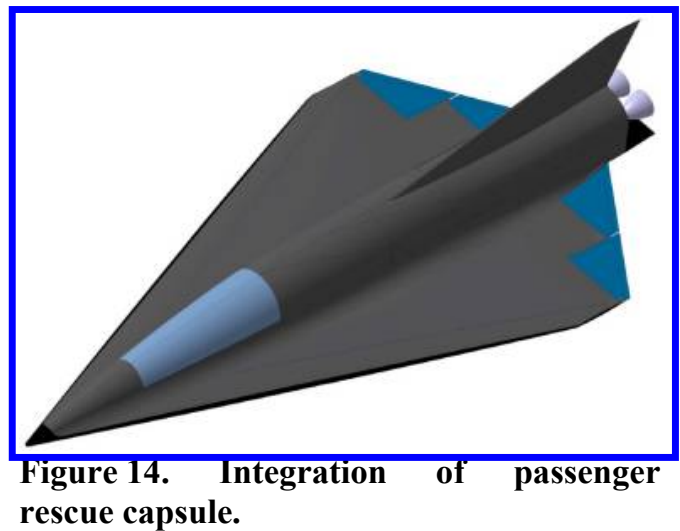

\section{B. Load Case Selection}

The structural design of a new aerospace vehicle requires the consideration of a large number of potential load cases, especially if the vehicle is unique in design and mission. A discussion of potential load cases for the SpaceLiner can be found in ref. 6. However, for trade studies on a preliminary level it is not appropriate to consider all potential load cases. Instead, a few number of cases should be selected that may be of major importance for sizing the vehicle structure. For the current analyses of the SpaceLiner orbiter, two load cases have been selected:

1) Maximum axial acceleration during booster assisted vertical ascent; while the orbiter is mounted on the booster at two axial stations

2) Maneuver with $2.5 \mathrm{~g}$ normal acceleration and empty propellant tanks

A $2.5 \mathrm{~g}$ normal acceleration maneuver is one of the most important load cases to be considered in order to fulfill civil aviation requirements ${ }^{15}$. For many aerospace vehicles maneuver load cases with full propellant tanks are the most critical. For the SpaceLiner however, all propellants, excluding residuals and reserves, will be completely consumed during ascent. Thus, the propellant tanks will be almost empty as soon as the gliding phase begins. For flight abort cases during ascent, with full tanks, an emergency propellant dumping procedure may be applied, where the details of such a safety feature have not yet been worked out. For both load cases aerodynamic pressure 
distributions have been provided by Hotsose. For the maneuver load case the inertia relief capability of Ansys is being exploited. Thus, accelerations in the axial and normal directions are affected by the applied load environment and the vehicle mass. Since the structural mass is not exactly known prior to the analysis, the actual normal accelerations may slightly differ from the envisaged $2.5 \mathrm{~g}$. Table 2 summarizes the load case data. The wall and stiffener thicknesses as computed in load case 1 will be set as minimum values for load case 2 . The introduction of flap/rudder loads into the orbiter wing structure, as shown in ref. 6 and ref. 16 have been neglected.

Table 2. Load case definition.

\begin{tabular}{|c|c|c|}
\hline & Load case 1 & Load case 2 \\
\hline Axial acceleration [g0] & 2.42 & $0.75-0.91$ \\
\hline Normal acceleration [g0] & 0.01 & $\approx 2.5$ \\
\hline Angle of attack [ ${ }^{\circ}$ ] & 0.2 & 19.2 \\
\hline Ma [-] & 3.8 & 10.0 \\
\hline Altitude [km] & 35.2 & 41.0 \\
\hline Static pressure LOX tank [bar] & 8.5 & 2.0 \\
\hline Static pressure LH2 tank [bar] & 2.5 & 2.0 \\
\hline Propellant mass [t] & 72.8 & 3.5 \\
\hline
\end{tabular}

The static tank pressures are increased by hydrostatic pressures that result from the particular accelerations. The static pressure for the LOX tank in load case 1 has been set to 8.5 bars in order to account for a propellant cross feeding system that has been envisaged between the booster and orbiter. The LOX tank of the booster is placed significantly forward of the LOX tank of the orbiter, yielding a corresponding hydrostatic pressure build-up through the feed lines. The general architecture of this system is depicted in Fig. 15. For load case 2 the tanks are almost empty, except of about $3.5 \mathrm{Mg}$ of residuals and reserves.

All sub-systems and payloads have been included as mass points that are attached to fuselage frames or wing rib/spar junctions.

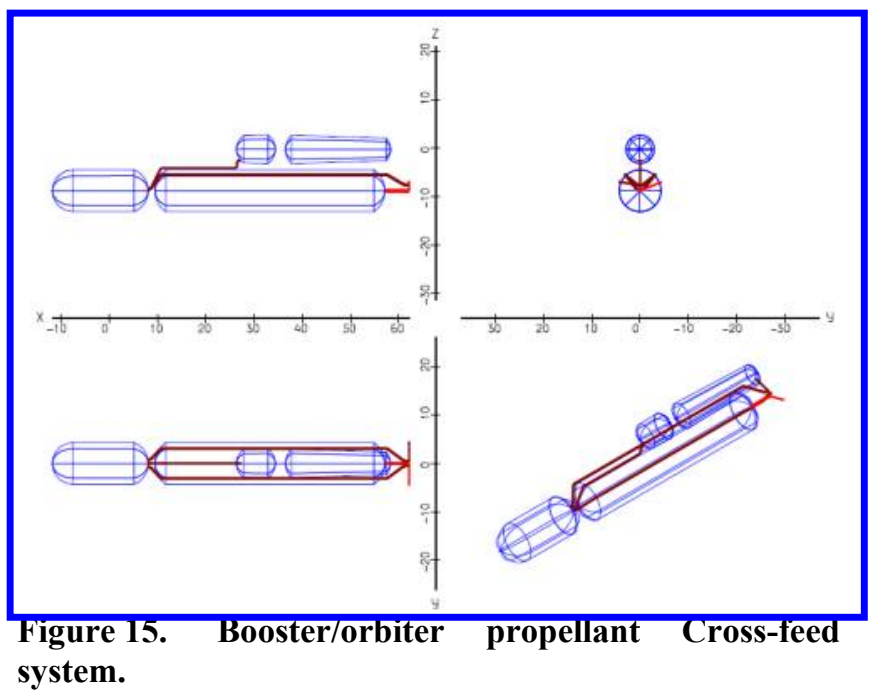

\section{Structural Analysis Trade-off Study Results}

As outlined previously, trade studies have been performed to allow for identification of tendencies and find reasonable solutions, without the expectation of an optimal result. In particular, the trade studies include:

1) Structural design concept studies

2) Material comparisons

3) Number/spacing of structural members

\section{Vehicle Geometry and Modelling}

The outer shape of the SpaceLiner orbiter has been generated within previous aerodynamic and aerothermodynamic design and optimization processes. Rescue stage and tank geometries as well as positions within the orbiter are fixed. The complex orbiter/passenger stage integration has not been reproduced in this analysis in order to reduce the model complexity. Instead, the stage remains completely encapsulated within the fuselage and is modeled as conical pressure vessel with 1 bar internal pressure.

Free geometric variables for the structural design are only positions of the structural members. However, the positions of some of the members have already been fixed and will not be changed. This includes fuselage frames at the beginning and ends of the tanks and the passenger stage, as well as at the booster attachment points and the main engine load introduction position. Furthermore, some wing rib and spar positions are fixed, due to, for example, the predetermined main gear positions. Part a) of Fig. 16 shows the geometry model with the fixed structural members 
only. Subsequently, additional members have been added as shown in part b) of the figure, whereas the fuselage frame/bulkhead spacing generally is not higher than $3 \mathrm{~m}$. The wing spars have a higher spacing. This geometry model forms the baseline configuration of the current investigations. The effect of varying the spacing of the structural members will be investigated later in this section. In fact, spacing of structural members is usually lower for real aircraft ${ }^{17}$.

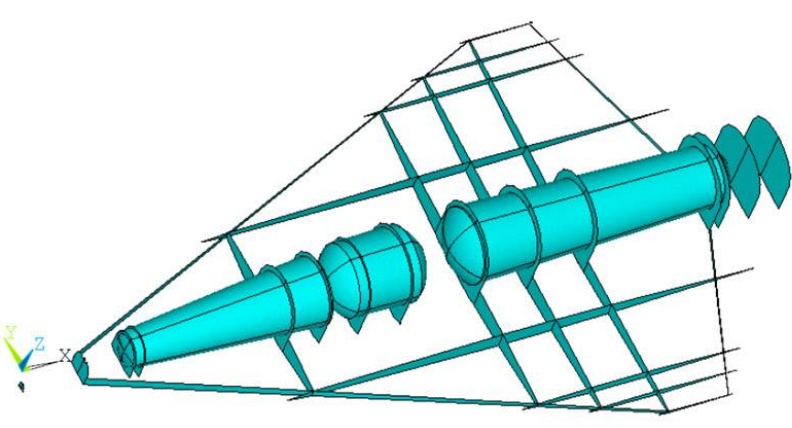

a)

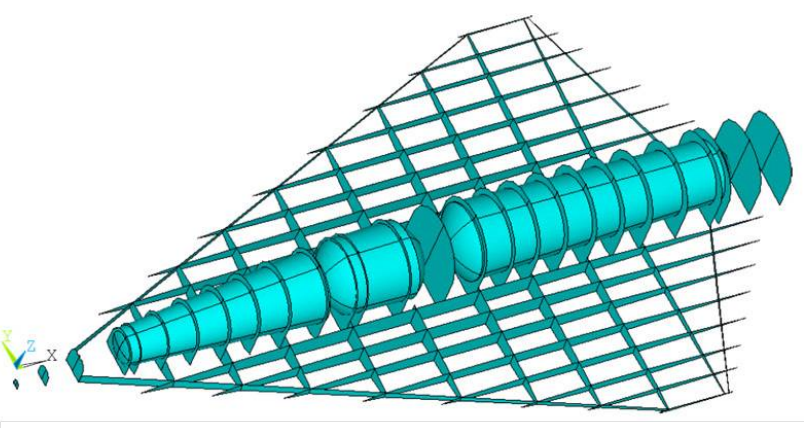

b)

Figure 16. Baseline configuration with skins removed; fixed members only a), all members b).

All structural members are modeled with shell elements. Fuselage frames/bulkheads may be intersected by tanks or the passenger stage. In this case, corresponding cut-outs will be generated in the bulkhead area and the remaining ring-frame will be connected completely to the frame. This means, that the tanks support the fuselage in carrying the bending loads. In fact, it has been decided to go for an approach where the tanks carry their own loads only, with the fuselage completely supporting the bending loads. However, this kind of design has not yet been modeled in HySAP and will not be considered within the trade studies presented here.

The fin of the vehicle has not been modeled since only symmetric load cases are being considered. Instead, it is considered as mass point, together with the other sub-systems.

\section{Structural Design Trade-off Study}

A total of six different design configurations have been investigated. Since wing, fuselage and tank stiffnesses may significantly influence each other (as for instance was found during the ATLLAS II study), the total configuration was investigated, even in the case where only the design of the wing or the fuselage had been changed. The baseline design is outlined in Table 3 . Table 4 lists the changes for each computation. For this study, the whole vehicle structure is made of Aluminum. The allowable stresses have been reduced by a safety factor of 1.5 .

Part a) of Fig. 17 shows the computed structural mass after load case 1, and part b) after successive consideration of load cases 1 and 2 . The propellant tanks are sized mainly by load case 1 due to the higher static and hydrostatic pressures. Fuselage and wing masses instead increase significantly after consideration of load case 2 (note the identical scaling of both parts of the figure). Configurations 2-5 are to be compared with the reference configuration 1 , rather than with each other. Particularly when comparing the first three runs, an interaction between wing and fuselage is identifiable (as seen similarly in the ATLLAS II study). If the wing becomes heavier and stiffer, the fuselage Table 3. Structural design baseline.

\begin{tabular}{|c|c|}
\hline Group & Design \\
\hline Wing ribs \& spars & unstiffened skin \\
\hline Wing skin & sandwich \\
\hline Fuselage skin & stringer stiffened skin \\
\hline Fuselage frames/bulkheads & sandwich \\
\hline Tank cylinders/cones & stringer stiffened skin \\
\hline Tank domes & unstiffened skin \\
\hline
\end{tabular}
mass decreases. It becomes clear, that an uniaxial stringer stiffening in span-wise direction (run 3) is not competitive. This is not a surprising result since the wing not only experiences strong span-wise, but also chord-wise bending, which has to be resisted by the skin alone. Run 2, with all wing members made of a sandwich material, yields the lowest structural mass. However, the wing structural mass is extraordinary low, which may be a result from limited implementation of failure criteria for sandwich

Table 4. Structural design variations.

\begin{tabular}{|c|c|}
\hline Calculation run & Variation (relative to baseline) \\
\hline 1 & Baseline \\
\hline 2 & Sandwich wing ribs \& spars \\
\hline 3 & Stringer stiffened wing skins \\
\hline 4 & Unstiffened fuselage frames \\
\hline 5 & Sandwich fuselage skin \\
\hline 6 & Unstiffened tank skins \\
\hline
\end{tabular}


materials. Except runs 2 and 3, the wing mass is similar to the fuselage mass. However, as described in ref. 6, the wing mass may increase if rudder/flap deflection or landing load cases are being considered.

For definition of useful structural design concepts, Fig. 17 reveals that sandwich design may be preferred for the total wing structure (run 2). For the fuselage instead sandwich design yields a higher structural mass than stringerstiffening (run 5). Additionally, unstiffened propellant tanks could yield a further slight mass decrease (run 6), as they are mainly sized by tensional loads due to high internal pressures.

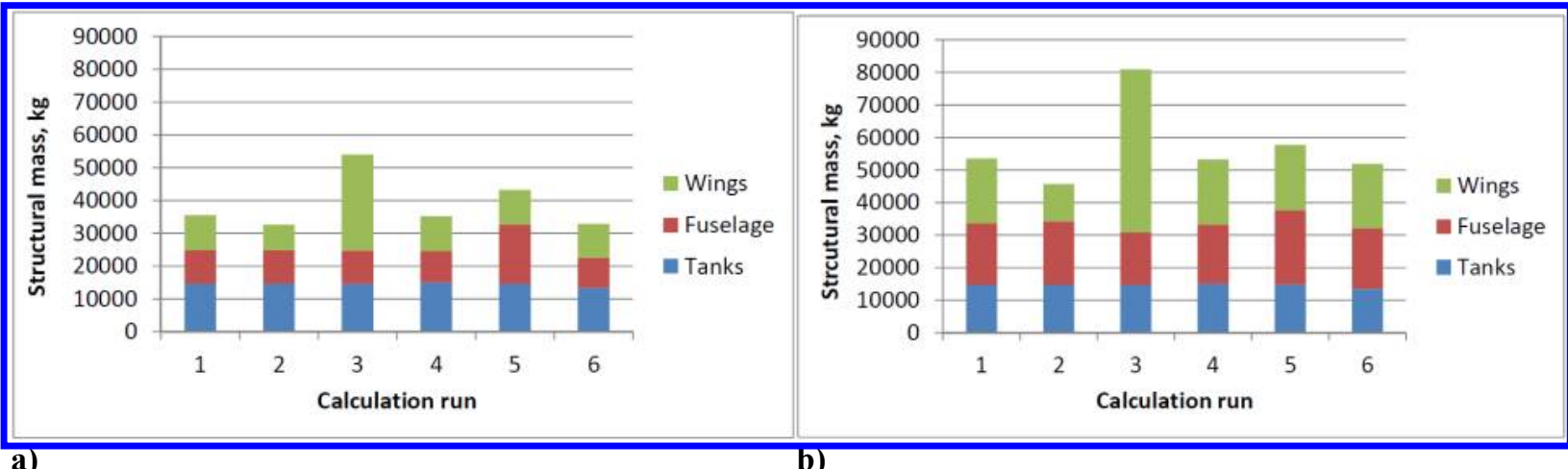

Figure 17. Structural masses for load case 1 a), and load case $1+2$ b).

Figure 18 a) shows the Von Mises stress distribution for the vehicle upper side from run 1, and Fig. 18 b) for the lower side. Both figures show the stress in the skin/facesheet layer of the vehicle when load case 2 is applied. As might be expected, the stresses on the upper side of the wing are comparatively low, since the upper side is mainly loaded in compression, and thus buckling critical. The lower wing side on the contrary, mainly faces tensile loads. Figure 19 shows a similar stress evaluation for the vehicle with the outer skins removed. Passenger capsule and LH2 tanks face comparatively high stress levels. The stresses in the LOX tank instead are low, since this tank is mainly sized by the significantly higher pressures in load case 1. As Fig. 18 and Fig. 19 indicate, the maximum stresses reach about $300 \mathrm{MPa}$, which is higher than the allowed stresses of $240 \mathrm{MPa}$. Closer inspection revealed that these higher stresses occur locally at some tank/bulkhead connections.
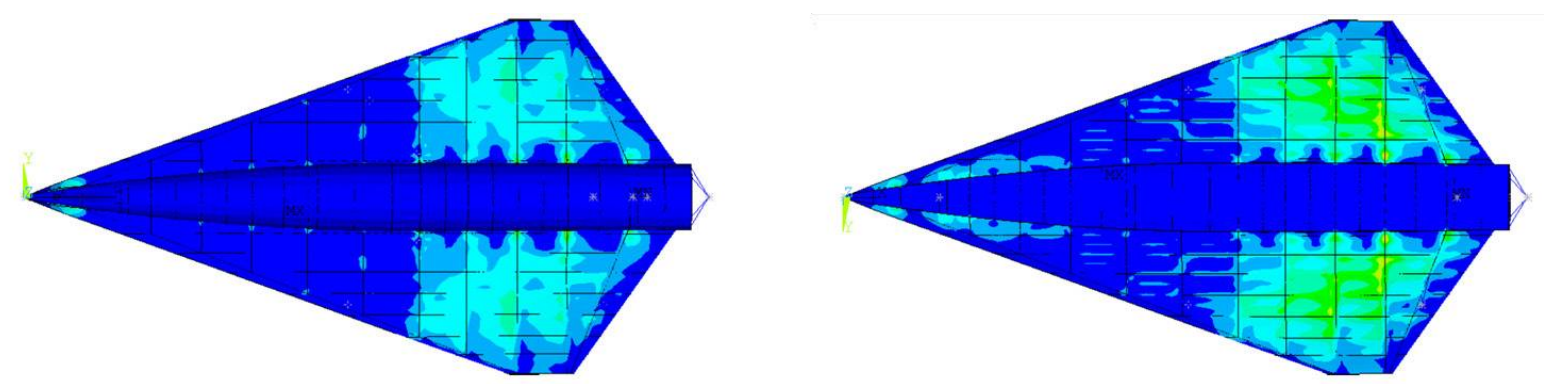

a) b)

Figure 18. Von Mises stress for configuration run 1 after finishing HySAP calculation for load case 2; stresses on the vehicle upper side a), and lower side b). 


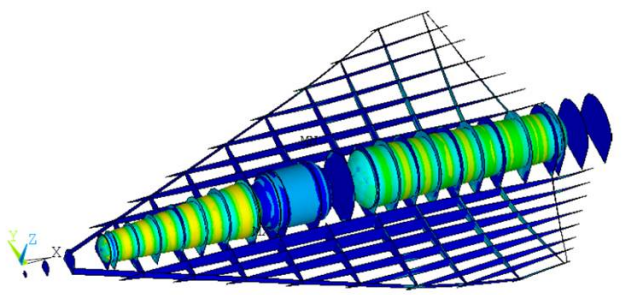

a)

Figure 19. Von Mises stress for configuration run 1 after finishing HySAP calculation for load case 2 with skins removed; stresses on the vehicle upper side a), and lower side b).

Fig 20 shows the stress distribution from Fig. 18 in another perspective to demonstrate the corresponding displacements. As expected, the wing is bended up-wards. The maximum displacement is $0.33 \mathrm{~m}$. Note the subsystem point masses in the vehicle aft section that are representing flaps, fin and fin sub-systems. Fuselage- or wing-internal sub-systems are not visible in this figure.

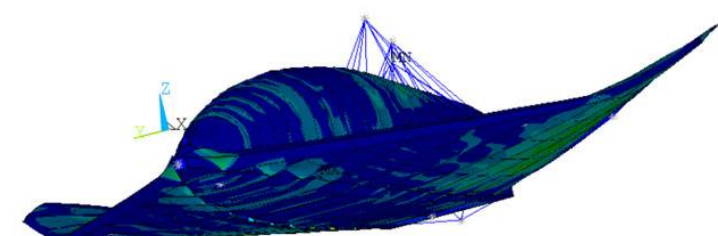
a) b)

Figure 21 demonstrates the convergence behavior of the HySAP adaption procedure. The structural mass for run 1 is shown for load case 1 and load case 2. After around 5-7 iterations the final mass value has approximately been reached.

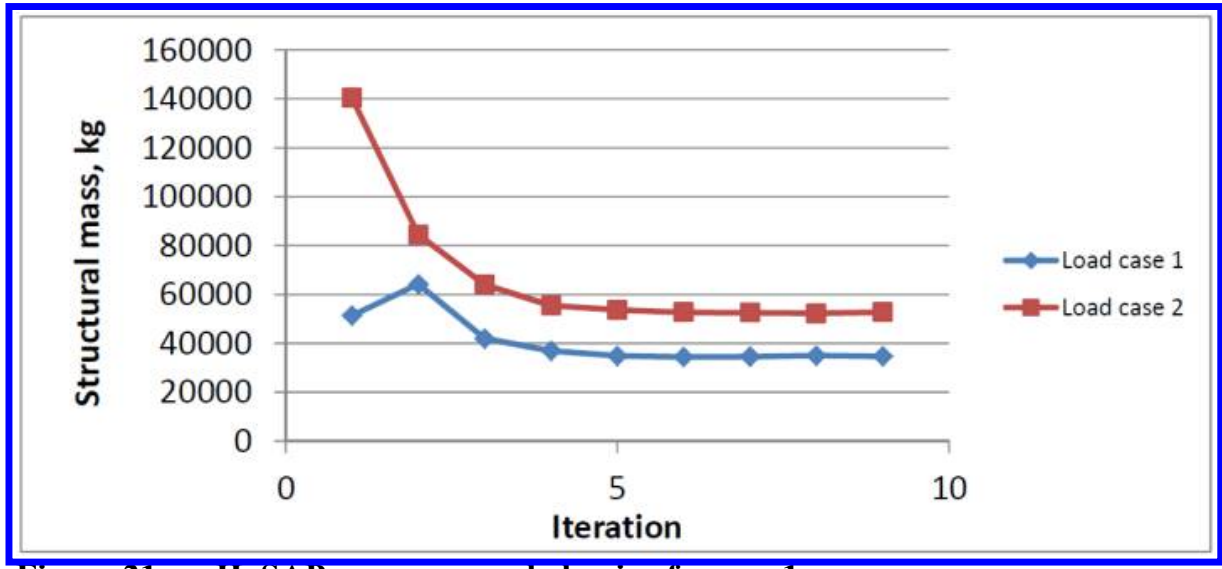

Figure 21. HySAP convergence behavior for run 1.

\section{Material Trade-off Study}

So far, only an all-aluminum and an all-Titanium structure have been compared, as shown in Table 5. Additional trade-off calculations are to be performed in the future. The structural design concepts correspond to the baseline configuration as outlined in the previous sub-section. The first calculation run is the baseline configuration with an all-aluminum structure. 
Table 5. Material trade-off.

\begin{tabular}{|c|c|}
\hline Calculation run & Materials \\
\hline 1 & All Al-2024 structure \\
\hline 7 & All Ti6Al-4V structure \\
\hline
\end{tabular}

Figure 22 shows the results. The Titanium vehicle is about $3.5 \mathrm{Mg}$ heavier than the Aluminum vehicle. The tanks however turned out to be lighter for Titanium since they are mainly sized by tensile loads, whereas the selected Titanium alloy offers a better strength to density ratio than the Aluminum alloy.

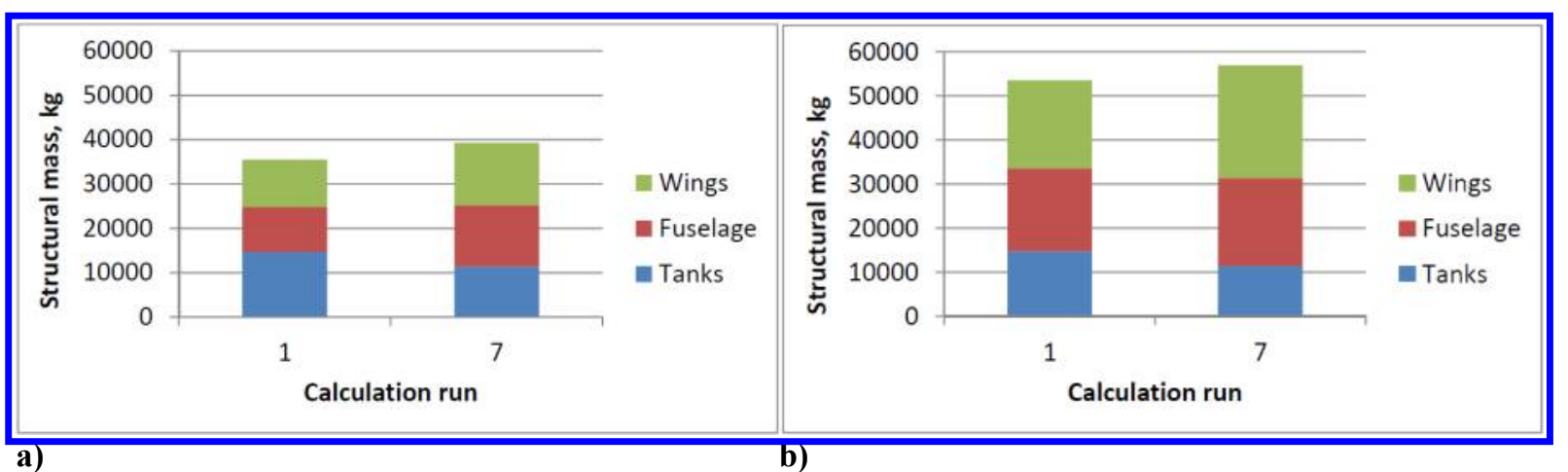

Figure 22. Aluminum/Titanium structural mass comparison for load case 1 a), and load case $1+2$ b).

The Titanium structure offers the advantage of higher structural temperatures, thus allowing for lower TPS masses. Therefore, it is appropriate to also evaluate the TPS weight. For the current design of the SpaceLiner7 no TPS mass comparison for different back-structure temperatures has been done so far. However, in ref. 6, a TPS mass comparison for a previous design of the SpaceLiner7 has been published. The results cannot be compared directly due to slightly different vehicle geometry and trajectory, but tendencies may be identified. According to the reference, for a back-structure temperature of $400 \mathrm{~K}$, which may be appropriate for Aluminum, the TPS becomes almost $10 \mathrm{Mg}$ heavier than for a back-structure temperature of $600 \mathrm{~K}$. This would more than outbalance the $3.5 \mathrm{Mg}$ heavier Titanium structure.

A corresponding TPS mass comparison for the current design of the SpaceLiner7 will be done in the near future. Nevertheless it is obvious, that structure and TPS mass have to be considered together in order to find low dry mass solutions for the orbiter.

\section{Variation of Number/Spacing of Structural Members}

The spacing of the structural members, such as ribs, spars or frames, has a large impact on structural masses. Generally, decreasing the spacing (and increasing the number) of the members may greatly reduce buckling vulnerability and increase the stiffness. On the other hand, additional members increase the total structural surface. This is particularly critical, if the wall thicknesses of the members approach the minimum gauge thickness. In fact, ref. 13 and ref. 18 argue that significant portions of an aerospace vehicle may be sized by minimum gauge requirements instead of load considerations. In this case increasing the number of the members will clearly increase the structural mass.

For identification of tendencies, two alternative wing rib/spar spacing concepts have been compared with the baseline design. Since the spacing for the baseline configuration was assumed to be already high, the two alternative designs utilize a smaller spacing, which yields higher number of ribs and spars. Figure 23 shows the three different configurations, with the baseline design at the left hand side. Note the irregular spacing between some of the members, governed by geometrical- and load introduction constraints (see sub-section 1.). 


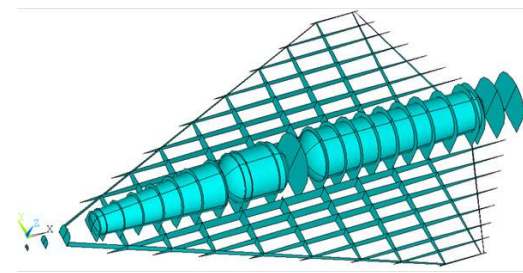

a)

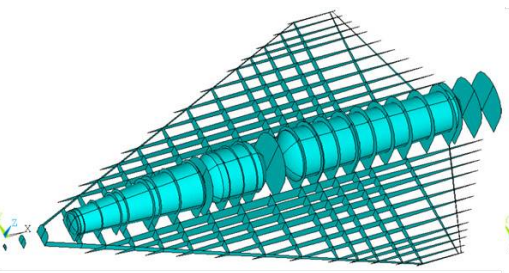

b)

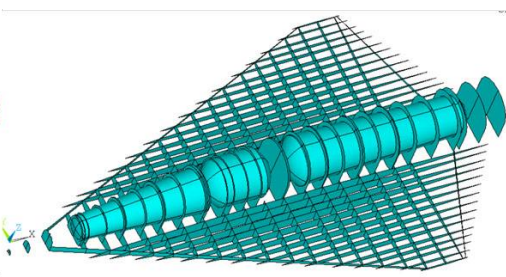

c)

Figure 23. Structural member arrangement for baseline a), run 8 b) and run 9 c) configuration

Figure 24 displays the results. Again, part a) presents the structural masses for load case 1 only, while part b) shows the masses after sizing for both load cases. As may be seen, the structural mass increases for higher number of ribs and spars with the baseline design offering the lightest mass.

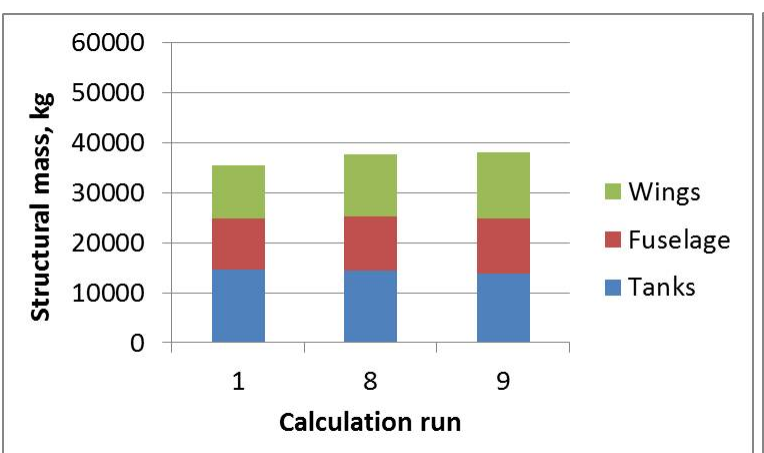

a)

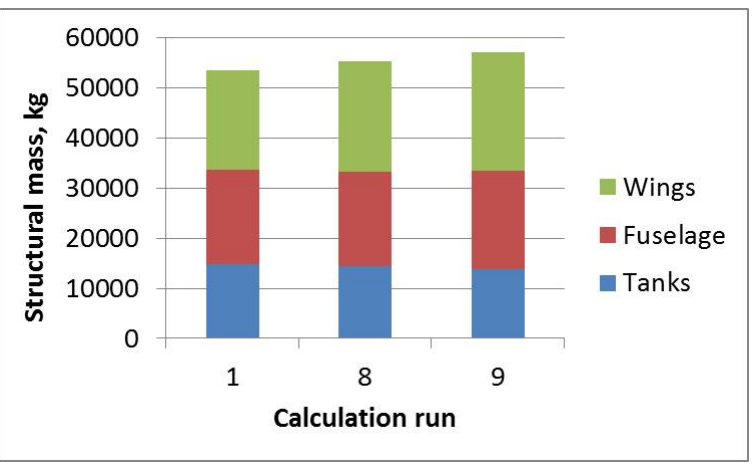

b)

It was found, that for the configurations with the higher rib and spar numbers the majority of the wing members has been sized down the minimum gauge thickness. Thus, no weight decrease could be achieved relative to the baseline configuration. In fact, the higher number of fuselage frames that are generated together with the spars yielded a decrease of the propellant tank masses. However, this tank mass decrease could not outbalance the higher wing mass.

\section{Estimation of TPS Thicknesses for the SpaceLiner7}

In order to determine the thickness of the TPS, the overall maximum temperatures and heat loads of nominal and abort trajectories have to be determined for the full vehicle surface. For this task, only fast engineering methods can be used. A preliminary flow analysis based on modified Newtonian surface inclination techniques is done while friction drag is estimated for each panel with the classical analytical methods for compressible laminar or turbulent flow of van Driest and White-Christoph. The surface temperatures are calculated under assumption of an adiabatic wall in radiation equilibrium. Heat fluxes are determined by using the Fay-Ridell equation close to the stagnation point and the Zoby-Moss-Sutton approach further downstream. The real gas effects on gas dynamic and transport properties can be considered in the calculation for chemically reacting air in equilibrium ${ }^{19}$.

Under the assumption of a fully turbulent flow, the heat fluxes are calculated for each mesh point at selected flight conditions along the different trajectories, with given Mach Number, angle of attack and altitude given by the trajectory simulation.

Optimizing the material thickness for each of the thousands of mesh points on the vehicle would be excessively computational intensive. Additionally, this would yield a design without sufficient margin on the TPS thickness and that would be unpractical for manufacturing. Therefore, the vehicle surface is divided into a number of different regions, depending on the overall maximum temperature for all nominal and abort trajectories (Fig. 25 and Fig. 26). 


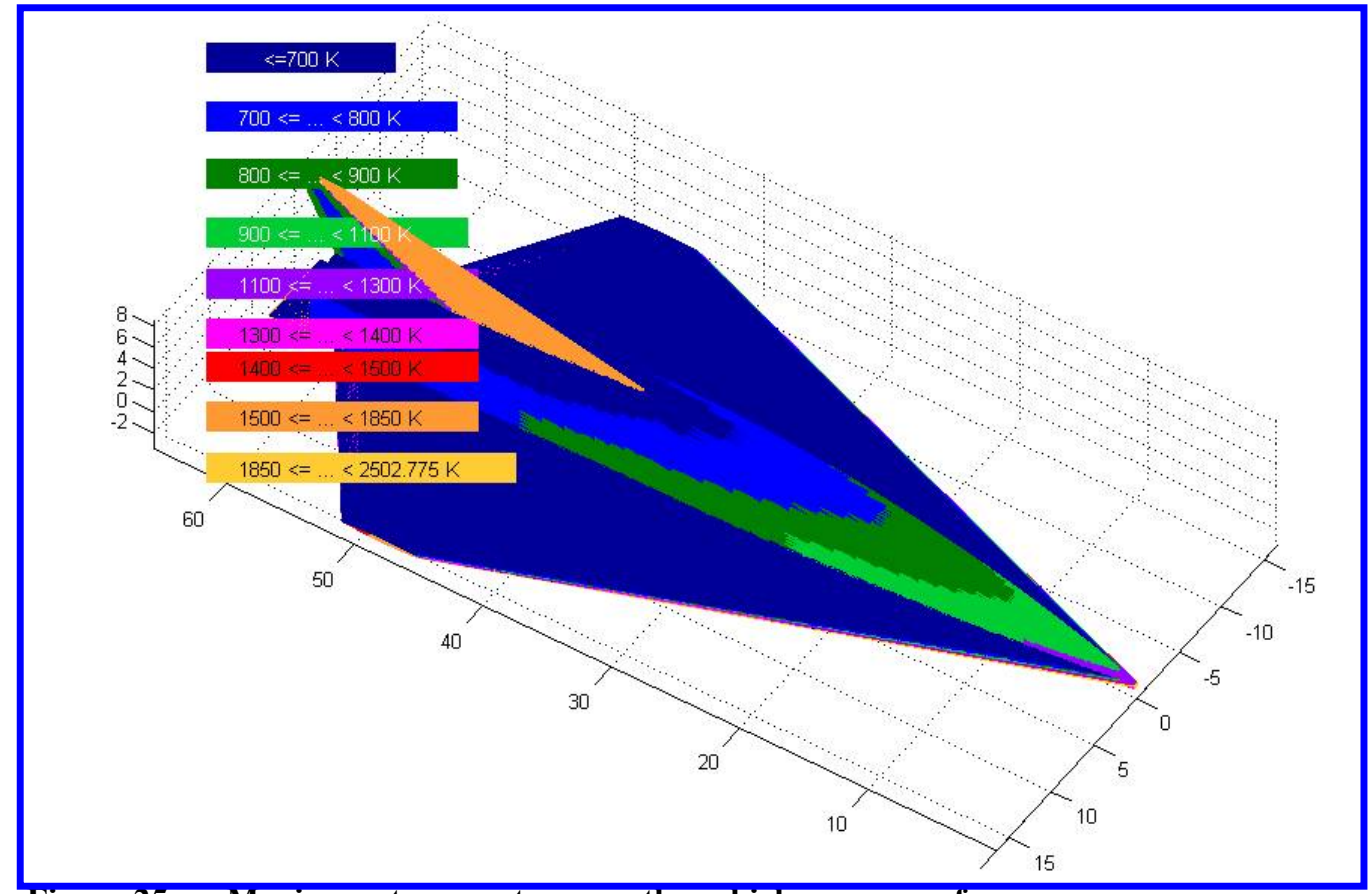

Figure 25. Maximum temperatures on the vehicle upper surface.

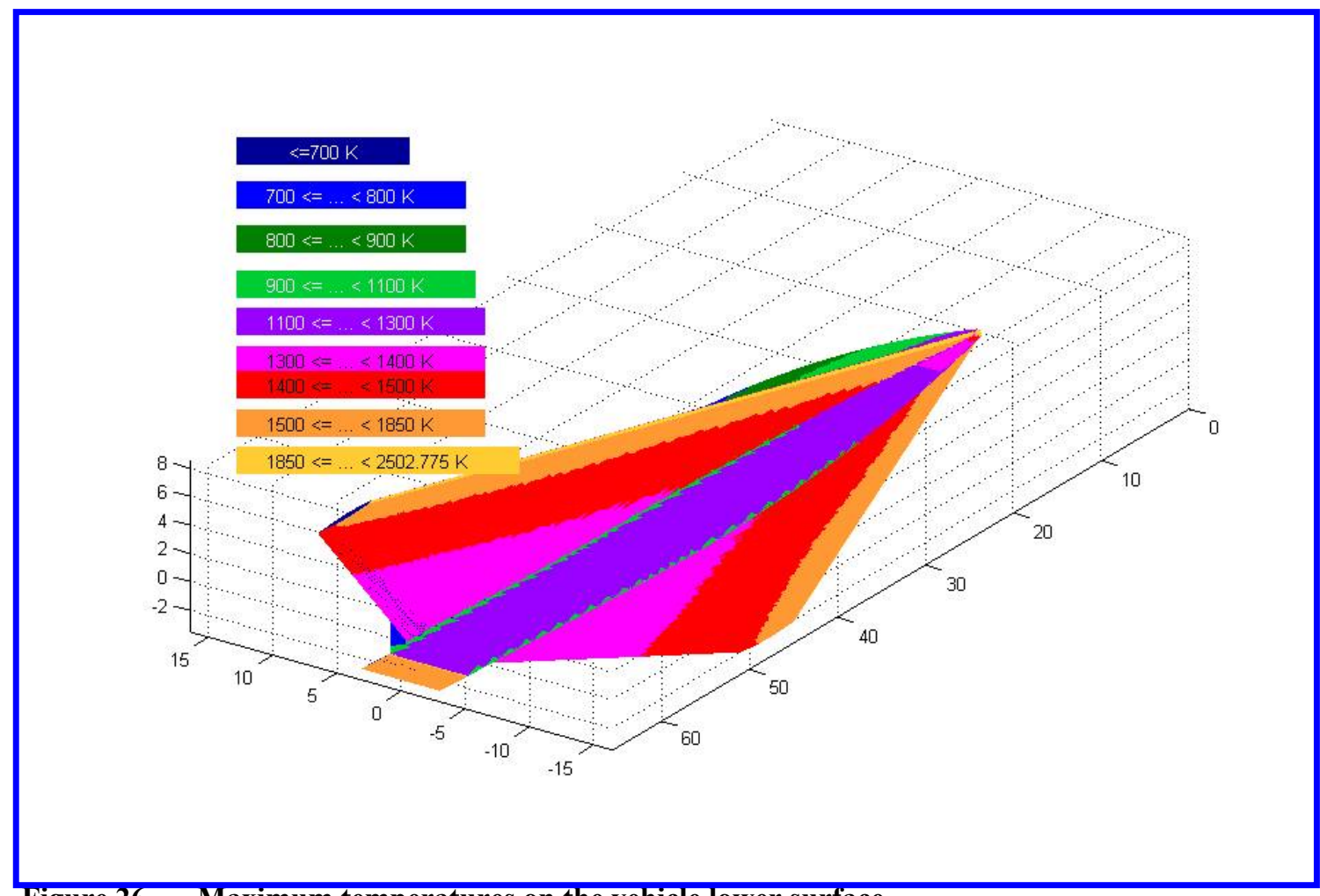

Figure 26. Maximum temperatures on the vehicle lower surface 
The maximum acceptable temperatures for the passive TPS is thereby limited to approximately $1850 \mathrm{~K}$ to be compliant with the reusability requirement. The structure is set to be allowed to heat up to $530 \mathrm{~K}$. This leads to a total TPS mass of approximately 21.6 tons. Details can be found in table 6 .

\begin{tabular}{|l|l|l|l|l|}
\hline Material & Temperature $[\mathrm{K}]$ & Thickness $[\mathrm{m}]$ & Coated surface $\left[\mathrm{m}^{2}\right]$ & Mass $[\mathrm{kg}]$ \\
\hline CMC & $1501-1850$ & 0.2396 & 47.25 & 935 \\
\hline CMC & $1401-1500$ & 0.2034 & 67.52 & 1250.5 \\
\hline CMC & $1301-1400$ & 0.1921 & 187.12 & 3391.7 \\
\hline CMC & $1101-1300$ & 0.1809 & 577.95 & 10248.2 \\
\hline CRI & $901-1100$ & 0.1434 & 175.63 & 2378.7 \\
\hline AFRSI & $801-900$ & 0.0454 & 70.45 & 398.6 \\
\hline AFRSI & $701-800$ & 0.0352 & 232.95 & 1088.8 \\
\hline AFRSI & $530-700$ & 0.0238 & 55.39 & 1991.2 \\
\hline \multicolumn{7}{|r|}{} & Total & $\mathbf{2 1 6 8 2 . 8}$ \\
\hline
\end{tabular}

Table 6. TPS thickness and mass breakdown.

The next step in further investigations will be to subtract the TPS thicknesses from the construction height of wing and fuselage. For this new contour, the structural analyses will be repeated. It may be expected, that the structural weight will increase when utilizing the new contour. It will be necessary to compare the thicknesses and mass of the TPS for different back-structure materials with the structural mass itself, to find a low overall mass solution.

\section{E. Structural-Dynamic and Aeroelastic analysis with NASTRAN for the SpaceLiner within FAST20XX}

Based on the structural sizing obtained with the HYSAP-tool, as described above, some further studies were performed at FOI for the orbiter using the software MSC-NASTRAN. In these studies the focus was on structural dynamics and aeroelastic considerations. The first fundamental vibration frequencies (eigen-frequencies) were calculated and examined. The first observation was the need for some local stiffness increase of the wing-beam webs to avoid low-frequency structural vibrations. The dimensions of structural members in the fuselage were then adjusted to obtain reasonable mode-shapes and frequencies. An observed flexibility of the fuselage cross-sections was reduced through design improvements. The first frequency, corresponding to fuselage bending, is $4.1 \mathrm{~Hz}$ with filled tanks. Without fuel, this frequency increases to $4.9 \mathrm{~Hz}$, and the wing-bending mode reaches $5.1 \mathrm{~Hz}$. This may be compared to $8.87 \mathrm{~Hz}$ for the wing when clamped to a rigid wall. The corresponding modes and frequencies obtained after an optimization, described below, can be seen in Fig. 27.

Stress-levels were calculated for a symmetric pull-up manoeuvre and some fairly local resizing was made. An analysis of pitch control authority for the ailerons was performed. The airflow is modelled using a panel method (DLM), widely utilized in the aerospace industry. The aerodynamic model is composed of 240 panels for the fuselage, and 432 for the wing. Control surfaces and ailerons are included to balance (trim) the aircraft. The "Surface SPLINE method" is used for linking different parts of the aircraft structure to the corresponding aerodynamic panels for aeroelastic analysis.

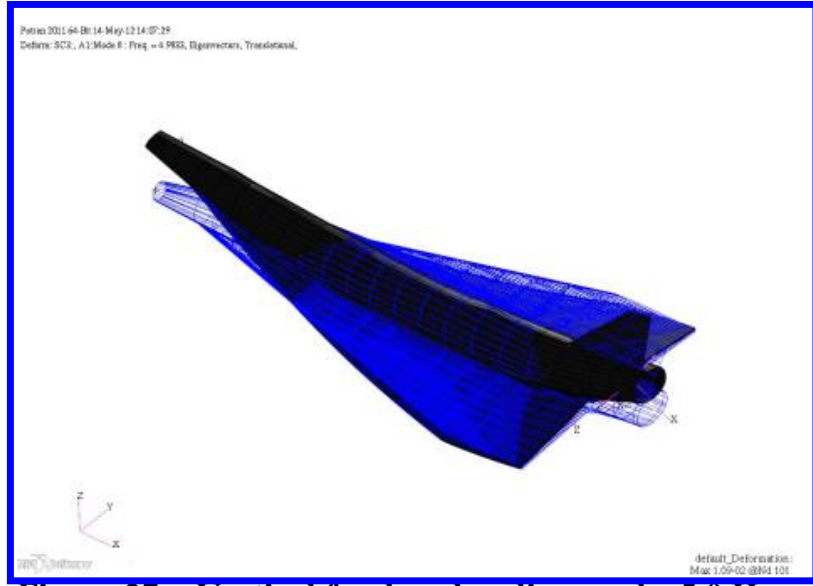

Figure 27a: Vertical fuselage bending mode. 5.0 Hz

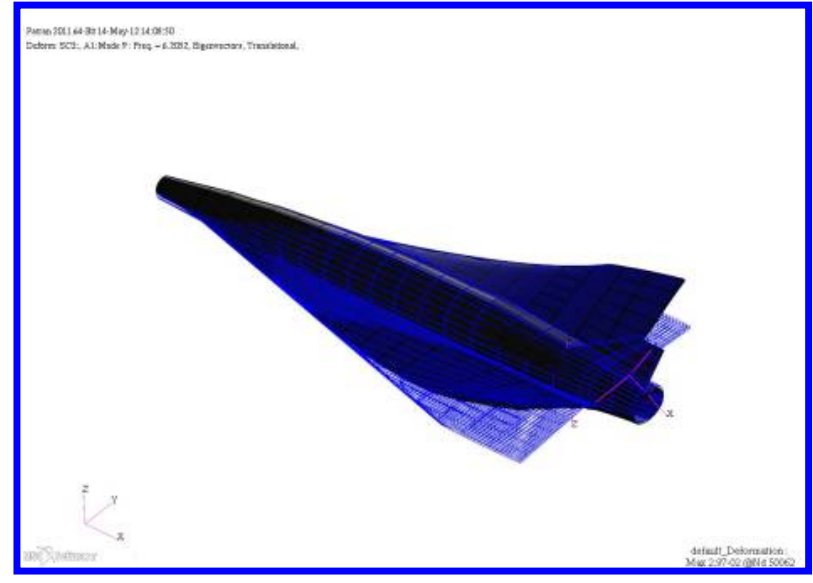

Figure 27b: Symmetric wing bending mode. $6.2 \mathrm{~Hz}$

18

American Institute of Aeronautics and Astronautics 
The optimization -method used is the MSC-Nastran SOL 200, and the Nastran sub-cases involved are:

- Quasi-aerostatic TRIM solution, SOL144, with stress analysis and aileron pitch performance,

- Modal response, SOL103,

- Flutter response, SOL145

The constraints for the optimization were in principle that neither maximum stress, eigen-frequencies, pitch control authority, nor flutter damping were allowed to deteriorate during the optimization. Furthermore, to avoid unrealistic dimensions, all design variables were constrained to the range [0.7-1.5] A total of 32 design variables, $d v$, where used. Structural dimensions relate to the corresponding design variable as follows: thickness- $d v$, area- $d v^{2}$, second moment of area $-d v^{4}$.

The results from the optimization indicate a potential for orbiter weight reduction of almost $9 \%$ of the lift-off weight. The only part of the vehicle where more structural mass was required was the region containing the rearmost wing-beam as may be seen in Fig. 28. It must however be remembered that adding more load-cases and the introduction of a large number of practical constraints will increase the structural mass compared to the calculated optima found here The stress-constraint (400 MPa) was also set at a very high value, especially in regions with compressive or shear-loading where buckling might require lower average stress-levels. Of the different constraints defined by the sub-cases there were no active constraints for the optimized design from the flutter response. This means that the flutter-damping is better than our requirement (constraint). For all the other sub-cases there were active constraints for the optimized design.

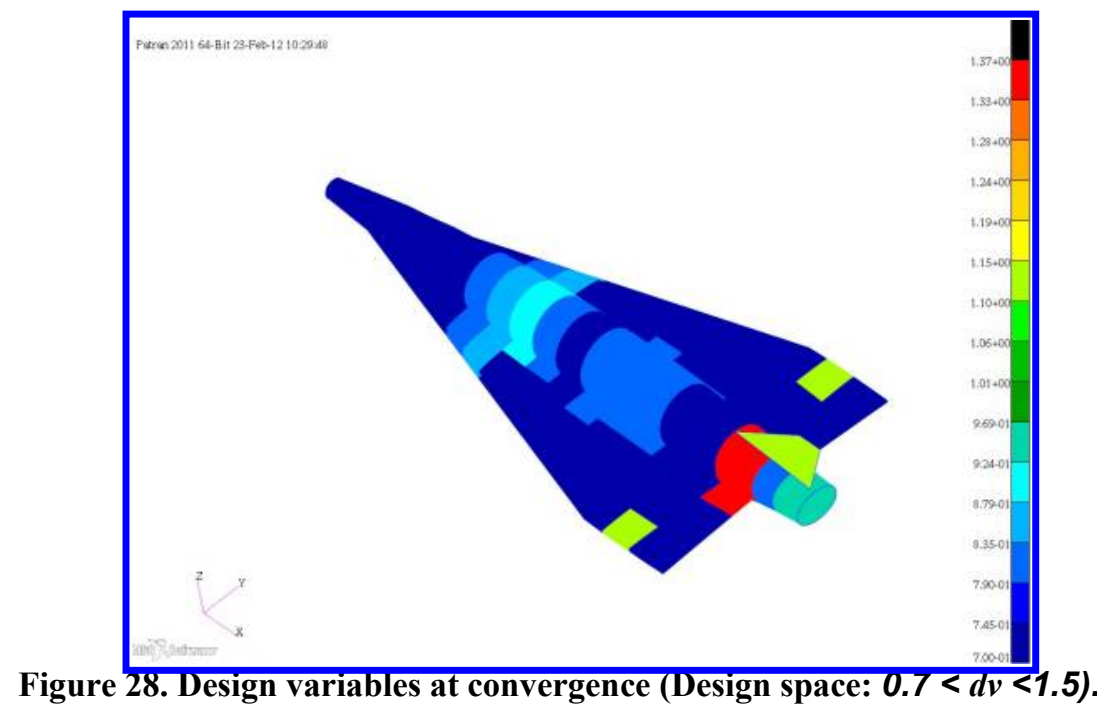

\section{Concluding Remarks}

The finite element based parametric structural analysis program HySAP has been described in detail. Parametric structural analysis for the SpaceLiner has been performed using HySAP with the results being presented. HySAP results from the recent ATLLAS II study have also been described, which altogether demonstrate the versatility of the tool. Furthermore, structural-dynamic and aero-elastic investigations have been performed to complement the static structural analysis as performed by HySAP.

The parametric structural study for the SpaceLiner is not finished, but initial results as presented here indicate that significant weight decrease may be possible by proper selection of structural concepts and materials as well as by consideration of integration of structure and TPS. More important, it has been shown that this kind of analysis can be performed already at an early design level, thus allowing for identification of design-drivers and for more precise predictions of the feasibility of highly mass critical, novel aerospace vehicle concepts. 


\section{Acknowledgments}

Part of this work has been performed within the FAST20XX, ATLLAS-II and CHATT projects.

FAST20XX 'Future High-Altitude High-Speed Transport 20XX' project is investigating high-speed transport systems, is coordinated by ESA-ESTEC, and supported by the EU within the 7th Framework Programme Theme7 Transport, Contract no.: ACP8-GA-2009-233816. Further information on FAST20XX can be found on http://www.esa.int/fast20xx.

ATLLAS-II 'Aero-Thermodynamic Loads on Lightweight Advanced Structures II' project is investigating highspeed air-breathing transport vehicles, is coordinated by ESA-ESTEC, and supported by the EU within the 7th Framework Programme Theme7 Transport, Contract no.: ACP1-GA-2011-285117.

Further information on ATLLAS II can be found on http://www.esa.int/techresources/atllas II.

CHATT 'Cryogenic $\underline{H} y$ personic $\underline{A}$ dvanced Tank Technologies' project is investigating tank technologies for high-speed transport, is coordinated by DLR-SART, and supported by the EU within the 7th Framework Programme Theme 7 Transport, Contract no.: ACP1-GA-2011-285117. Further information on CHATT can be found on http://www.chatt.aero.

\section{References}

${ }^{1}$ Sippel, M. et al.: Comparative Study on Options for High-Speed Intercontinental Passenger Transports: Air-Breathing- vs. Rocket-Propelled, IAC-05-D2.4.09, October 2005

${ }^{2}$ Sippel, M.: Introducing the SpaceLiner Vision, $7^{\text {th }}$ International Symposium on Launcher Technologies, Barcelona, April 25,2007

${ }^{3}$ Sippel, M., van Foreest, A.: SpaceLiner Rocket-Powered High-Speed Passenger Transportation Concept Evolving in FAST20XX, IAC-10-D2.4.06, Prague, September 2010

${ }^{4}$ Sippel, M., Schwanekamp, T., Bauer, C., Garbers, N., van Foreest, A., Tengzelius, U., Lentsch, A.: Technical Maturation of the SpaceLiner Concept, $18^{\text {th }}$ AIAA International Space Planes and Hypersonic Systems and Technologies Conference, Tours, France, September 2012, to be published 2011

${ }^{5}$ Tong Minh, B.: Design of the SpaceLiner Thermal Protection System, DLR-SART-Technical-Note-002/2011, Bremen,

${ }^{6}$ Kopp, A., van Foreest, A., Sippel, M., Dalenbring, M., Jarlas, R.: Investigation of Structure for the Hypersonic Transport System SpaceLiner; AIAA-2011-2373, 17th AIAA International Space Planes and Hypersonic Systems and Technologies Conference, San Francisco, 2011

${ }^{7}$ Johnson, F.T.: A General Panel Method for the Analysis and Design of Arbitrary Configurations in Incompressible Flows, NASA Contractor Report 3079, Boeing Company, Seattle, 1980

${ }^{8}$ Ciampa, P. D., Nagel, B., Van Tooren, M.: "Global Local Structural Optimization of Transportation Aircraft Wings", 51st AIAA/ASME/ASCE/AHS/ASC Structures, Structural Dynamics, and Materials Conference, Orlando, Florida, April 2010

${ }^{9}$ Ciampa, P. D.: "Global-Local Optimization of Stringer Stiffened Panels for Transportation Aircraft Wings", MSc Thesis, Faculty of Aerospace Engineering, Delft University of Technology, 2009

${ }^{10}$ Bruhn, E.F.: Analysis and Design of Flight Vehicle Structures. Tri-State Offset Company, USA, 1973

${ }^{11}$ Sullins, R. T., Smith, G. W., Spier, E. E.: Manual for Structural Stability Analysis of Sandwich Plates and Shells, NASA Contractor Report 1457, General Dynamics Corporation, San Diego, December 1969

${ }^{12}$ Ley, R. P., Lin, W., Mbanefo, U.: Facesheet Wrinkling in Sandwich Structures, NASA Contractor Report 1999-208994, Northrop Grumman Corporation, El Segundo, California, USA, January 1999

${ }^{13}$ Ardema, M. D.: Body Weight of Hypersonic Aircraft: Part 1, NASA Technical Memorandum 101028, Ames Research Center, Moffet Field, California, October 1988

${ }^{14}$ Zanchetta, M., Walton, C.: ATLLAS II Preliminary Vehicle Definition and Performance, ATLLAS II Deliverable D2.1.1, Gas Dynamics Ltd, Farnborough, August 2012

${ }^{15}$ European Aviation Safety Agency, "Certification Specifications for Large Aeroplanes CS-25", Amendment 2, October 2006

${ }^{16}$ Dietlein, I., Schwanekamp, T., Kopp, A.: Trim Requirements and Impact on Structural and Wing Design for the High Speed Passenger Transport Concept SpaceLiner, $4^{\text {th }}$ European Conference for Aerospace Science (EUCASS), St Petersburg, Russia, June 2011

${ }^{17}$ Sensmeier, M. D., Samareh, J. S.: “A Study of Structural Layouts in Post-WWII Aircraft", 45 AIAA/ASME/ASCE/AHS/ASC Structures, Structural Dynamics and Materials Conference, Palm Springs, California, April 2004

${ }^{18}$ Ardema, M. D.: Structural Weight Analysis of Hypersonic Aircraft, NASA Technical Note D-6692, Moffett Field, California, March 1972

${ }^{19}$ Sippel, M., Klevanski, J., van Foreest, A., Gülhan, A., Esser, B., Kuhn, M.: The SpaceLiner Concept and its Aerothermodynamic Challanges, 1st ARA-Days, Arcachon, July 2006 